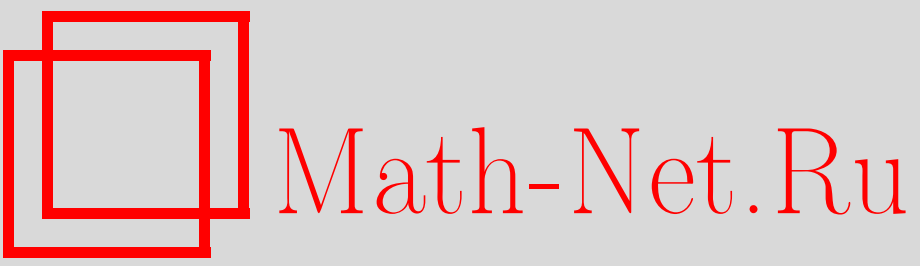

В. Е. Горюнов, М. М. Преображенская, Квазиустойчивость сосуществующих аттракторов нейродинамической модели с запаздыванием, Итоги науки и техн. Сер. Соврем. мат. и ее прил. Темат. обз., 2019, том 173, 26-47

DOI: https://doi.org/10.36535/0233-6723-2019-173-26-47

Использование Общероссийского математического портала Math-Net.Ru подразумевает, что вы прочитали и согласны с пользовательским соглашением

http://www.mathnet.ru/rus/agreement

Параметры загрузки:

IP: 34.229 .45 .116

26 апреля 2023 г., 08:35:54 


\title{
КВАЗИУСТОЙЧИВОСТЬ СОСУЩЕСТВУЮЩИХ АТТРАКТОРОВ НЕЙРОДИНАМИЧЕСКОЙ МОДЕЛИ С ЗАПАЗДЫВАНИЕМ
}

\author{
(c) 2019 г. $\quad$ В. Е. ГОРЮНОВ, М. М. ПРЕОБРАЖЕНСКАЯ
}

\begin{abstract}
АннотАция. Рассмотрен вопрос о сосуществовании квазиустойчивых аттракторов нейродинамической модели с запаздыванием, представляющей собой систему двух специальным образом связанных дифференциально-разностных уравнений. Разработан алгоритм оценки показателей Ляпунова для рассматриваемой системы.
\end{abstract}

Ключевые слова: дифференциально-разностное уравнение, буферность, релаксационный цикл, показатель Ляпунова, квазиустойчивость.

\section{QUASI-STABILITY OF COEXISTING ATTRACTORS OF A NEURODYNAMIC MODEL WITH DELAY}

\author{
(c) 2019 V. E. GORYUNOV, M. M. PREOBRAZHENSKAYA
}

\begin{abstract}
The problem of the coexistence of quasi-stable attractors of a neurodynamic model with delay is considered. The model is a system of two specially connected differential-difference equations. An algorithm for estimating Lyapunov exponents for the system is developed.

Keywords and phrases: differential-difference equation, buffering, relaxation cycle, Lyapunov exponent, quasi-stability.
\end{abstract}

AMS Subject Classification: 34D05, 34D08, 34D45, 65K05

1. Введение. При исследовании модели поведения двух нейроподобных осцилляторов с запаздывающей синаптической связью представляют интерес существование и устойчивость релаксационных циклов. Такая пара взаимодействующих нейронов моделируется следующими дифференциально-разностными уравнениями:

$$
\begin{aligned}
& \dot{u}_{1}=\left(\lambda f\left(u_{1}(t-1)\right)+b g\left(u_{2}(t-h)\right) \ln \frac{u_{*}}{u_{1}}\right) u_{1}, \\
& \dot{u}_{2}=\left(\lambda f\left(u_{2}(t-1)\right)+b g\left(u_{1}(t-h)\right) \ln \frac{u_{*}}{u_{2}}\right) u_{2} .
\end{aligned}
$$

Данная феноменологическая модель основана на идее быстрой пороговой модуляции (fast threshold modulation, см. $[20,21])$ и является модификацией модели, предложенной в [5]. В отличие от модели в [5], система (1.1) содержит дополнительное запаздывание $h>1$ в цепи связи. Здесь $u_{1}(t), u_{2}(t)>0$ - нормированные мембранные потенциалы нейронов. Каждый нейрон моделируется сингулярно возмущенным дифференциально-разностным уравнением

$$
\dot{u}=\lambda f(u(t-1)) u
$$

Работа выполнена при поддержке Российского фонда фундаментальных исследований (проект № 18-29-10055). 
из [12] (о нейромоделях с одним запаздыванием см. также [10]). Параметр $\lambda \gg 1$ характеризует скорость протекания электрических процессов, функция $f(u) \in C^{2}\left(\mathbb{R}_{+}\right)$, где $\mathbb{R}_{+}=\{u \in \mathbb{R}: u \geqslant 0\}$, такова, что

$$
f(0)=1 ; \quad f(u)+a, u f^{\prime}(u), u^{2} f^{\prime \prime}(u)=O\left(\frac{1}{u}\right) \quad \text { при } \quad u \rightarrow+\infty, \quad a=\text { const }>0 .
$$

Слагаемое $b g\left(u_{j-1}(t-h)\right) \ln \left(u_{*} / u_{j}\right) u_{j}$ в (1.1) моделирует синаптическое взаимодействие. Связь предполагается пороговой с запаздыванием $h>1$ по времени. Параметр $b=$ const $>0, u_{*}=$ $\exp (c \lambda)$ - пороговое значение, управляющее взаимодействием, $c=$ const $\in \mathbb{R}$.

Функция $g(u) \in C^{2}\left(\mathbb{R}_{+}\right)$удовлетворяет следующим условиям:

$$
\forall u>0 g(u)>0, \quad g(0)=0 ; \quad g(u)-1, u g^{\prime}(u), u^{2} g^{\prime \prime}(u)=O\left(\frac{1}{u}\right) \quad \text { при } u \rightarrow+\infty .
$$

В [12] показано, что при всех $\lambda \gg 1$ уравнение (1.2) допускает экспоненциально орбитально устойчивый релаксационный цикл $u_{*}(t, \lambda)>0$ периода $T_{*}(\lambda)$, удовлетворяющий предельным соотношениям

где

$$
\lim _{\lambda \rightarrow \infty} T_{*}(\lambda)=T_{0}, \quad \max _{0 \leqslant t \leqslant T_{*}(\lambda)}\left|x_{*}(t, \lambda)-x_{0}(t)\right|=O\left(\frac{1}{\lambda}\right), \quad \lambda \rightarrow \infty
$$

$$
T_{0} \stackrel{\text { def }}{=} 2+a+\frac{1}{a}, \quad t_{0} \stackrel{\text { def }}{=} 1+\frac{1}{a},
$$

$x_{*}(t, \lambda)=\ln \left(u_{*}(t, \lambda)\right) / \lambda$, а $T_{0}$-периодическая функция $x_{0}(t)$ задается равенствами

$$
x_{0}(t) \stackrel{\text { def }}{=}\left\{\begin{array}{ll}
t & \text { при } 0 \leqslant t \leqslant 1, \\
1-a(t-1) & \text { при } 1 \leqslant t \leqslant t_{0}+1, \\
t-T_{0} & \text { при } t_{0}+1 \leqslant t \leqslant T_{0},
\end{array} \quad x_{0}\left(t+T_{0}\right) \equiv x_{0}(t) .\right.
$$

Отметим, что в задачах, подобных (1.1) (и (1.2)), уместно сделать экспоненциальную замену (cм. [11])

$$
u_{j}=\exp \left(\lambda x_{j}\right), \quad j=1,2,
$$

которая позволит перейти к следующей системе с более содержательным предельным объектом:

$$
\begin{aligned}
& \dot{x}_{1}=f\left(\exp \left(\lambda x_{1}(t-1)\right)\right)+b\left(c-x_{1}\right) g\left(\exp \left(\lambda x_{2}(t-h)\right)\right), \\
& \dot{x}_{2}=f\left(\exp \left(\lambda x_{2}(t-1)\right)\right)+b\left(c-x_{2}\right) g\left(\exp \left(\lambda x_{1}(t-h)\right)\right) .
\end{aligned}
$$

При устремлении $\lambda$ к бесконечности правые части (1.8) стремятся к релейным функциям:

$$
\lim _{\lambda \rightarrow \infty} f(\exp (\lambda x))=R(x) \stackrel{\text { def }}{=}\left\{\begin{array}{ll}
1, & x \leqslant 0, \\
-a, & x>0,
\end{array} \quad \lim _{\lambda \rightarrow \infty} g(\exp (\lambda x))=H(x) \stackrel{\text { def }}{=} \begin{cases}0, & x \leqslant 0 \\
1, & x>0 .\end{cases}\right.
$$

Тогда в качестве предельного объекта модели (1.1) можно рассматривать релейную систему

$$
\begin{aligned}
& \dot{x}_{1}=R\left(x_{1}(t-1)\right)+b\left(c-x_{1}\right) H\left(x_{2}(t-h)\right), \\
& \dot{x}_{2}=R\left(x_{2}(t-1)\right)+b\left(c-x_{2}\right) H\left(x_{1}(t-h)\right) .
\end{aligned}
$$

В $[4-6,8,14]$ для систем, подобных (1.1) (с произвольным числом осцилляторов и без запаздывания $h$ в цепи связи), показано существование дискретных бегущих волн. Система (1.1) рассматривалась в $[3,15,18]$, где для нее исследовался вопрос существования и устойчивости специальных релаксационных периодических режимов: импульсно-рефрактерного (см. [15]), однородного (см. [3]) и противофазного (см. [18]).

В следующем разделе (п. 2) сформулированы необходимые определения и коротко описаны полученные результаты, касающиеся решений с несколькими всплесками на периоде.

В п. 3 исследовано более сложное поведение динамической системы, а именно, для релейной системы (1.9) аналитически доказано существование буферности, под которой понимается сосуществование у системы однотипных устойчивых решений и механизма их накопления. 
Раздел 4 посвящен вопросам выявления феномена квазиустойчивости различных режимов рассматриваемой модели. Понятие квазиустойчивости для моделей биологических ассоциаций было введено в [9]; в этой работе под квазиустойчивостью понимается наличие в спектре устойчивости релаксационного цикла наряду с единичным мультипликатором еще одного или нескольких, асимптотически близких по модулю к единице. В нашем случае мультипликаторы изучаемых циклов также оказываются близки по модулю к единице, хотя для них не выполняется условие асимптотического стремления к ней. Тем не менее, такие циклы мы тоже будем считать квазиустойчивыми.

2. Формулировка основных аналитических результатов. Рассмотрим вопрос о сосуществовании релаксационных циклов системы (1.8) с некоторым наперед заданным числом промежутков положительности на периоде. Отметим, что в силу экспоненциальной связи (1.7) функций $u_{j}$ и $x_{j}$ этим участкам положительности соответствуют асимптотически высокие всплески на периоде решения системы (1.1). Иначе говоря, в этом разделе мы будем заниматься поиском различных режимов, обладающих bursting-эфффектом-специальным свойством решения, характерным для нейродинамических систем (см. $[7,19])$.

Определение 2.1. Bursting-эффект - чередование нескольких подряд идущих высокоамплитудных всплесков, сменяющихся асимптотически малыми значениями мембранного потенциала.

Начнем с описания простейшего режима системы (1.8). Дадим определение соответствующего решения системы (1.1).

Определение 2.2. Назовем периодическое решение системы (1.1) импульсно-рефрактерным, если одна из функций $u_{1}(t), u_{2}(t)$ претерпевает периодические асимптотически высокие всплески, а другая при этом асимптотически мала при всех значениях $t>0$.

Введем следующие обозначения для констант:

$$
\xi \stackrel{\text { def }}{=} \exp \left(-b t_{0}\right), \quad \eta \stackrel{\text { def }}{=} \frac{a+1}{1-\xi}+\frac{1}{b}+c .
$$

Следующая теорема, доказанная в [15], означает наличие импульсно-рефрактерного режима у системы (1.1).

Теорема 2.1. Для каждого $q>0$, для которого выполнено неравенство

$$
\eta<-q,
$$

существует такое достаточно малое число $\varepsilon_{0}>0$, что при каждом $\varepsilon \in\left(0, \varepsilon_{0}\right]$ система $(1.8)$ допускает экспоненциально орбитально устойчивое решение $\left(x_{1}(t, \lambda), x_{2}(t, \lambda)\right)^{T}$ периода $T(\lambda)$, удовлетворяющее предельным равенствам

$$
\begin{gathered}
\lim _{\lambda \rightarrow \infty}\left(\max _{0 \leqslant t \leqslant T(\lambda)}\left|x_{1}(t, \lambda)-x_{0}(t)\right|+\max _{0 \leqslant t \leqslant T(\lambda)}\left|x_{2}(t, \lambda)-y_{0}(t)\right|\right)=0, \quad \lim _{\lambda \rightarrow \infty} T(\lambda)=T_{0}, \\
y_{0}(t) \stackrel{\text { def }}{=}\left\{\begin{array}{ll}
e(t), & h \leqslant t \leqslant t_{0}+h, \\
t-t_{0}-h+e\left(t_{0}+h\right), & t_{0}+h \leqslant t \leqslant T_{0}+h,
\end{array} \quad y_{0}\left(t+T_{0}\right) \equiv y_{0}(t),\right.
\end{gathered}
$$

гde

$$
e(t) \stackrel{\text { def }}{=} \frac{a+1}{1-\xi} \exp (-b(t-h))+\frac{1}{b}+c .
$$

Периодическая функция $x_{0}$ меняет знак, причем на периоде она обладает одним промежутком положительности и одним промежутком отрицательности, а функция $y_{0}$ при этом всюду отрицательна. В силу экспоненциальной зависимости $u_{j}(j=1,2)$ от функций $x_{j}$, близких к $x_{0}$ и $y_{0}$, это означает, что при больших $\lambda$ функция $u_{1}$ обладает на периоде асимптотически высоким всплеском, а $u_{2}$ асимптотически мала. Таким образом, решению, описанному в теореме 2.1, соответствует импульсно-рефрактерный режим системы (1.1). Отметим, что в силу симметричного вхождения неизвестных функций $u_{1}, u_{2}$ в систему (1.1) из теоремы 2.1 следует, что (1.1) обладает двумя импульсно-рефрактерными режимами.

Далее, перейдем к описанию синхронных режимов систем (1.1) и (1.8). 
Определение 2.3. Назовем периодическое решение системы (1.1) синхронныц, если функция $u_{2}(t)$ получается из $u_{1}(t)$ сдвигом по времени: $u_{1}(t)=u(t), u_{2}(t)=u(t+\Delta)$.

Определение 2.4. Синхронное решение системы (1.1) назовем однородным (синфазным), если $\Delta=0$.

Определение 2.5. Синхронное решение системы (1.1) назовем противофазным, если $\Delta=$ $T / 2$, где $T$ - период функции $u(t)$ (см. [21]).

Синхронные режимы системы (1.8) определяются аналогично.

В $[3,18]$ получен следующий результат, означающий существование у системы (1.1) устойчивых однородного и противофазного режимов с bursting-эффектом.

Теорема 2.2. Зафиксируем натуральное число l. Пусть

$$
\Delta=\frac{1}{3-\xi^{l}}\left((h-\eta)\left(1-\xi^{l}\right)+l T_{0}\left(1+\xi^{l}\right)\right),
$$

параметры $a, b, c, h$ удовлетворяют ограничениям

$$
a+\eta<0, \quad t_{0}+1+(2 l-1) T_{0}-\frac{1}{2}\left(1-\xi^{l}\right)(\eta+a)<h<2 l T_{0} .
$$

Тогда система (1.8) одновременно обладает экспоненииально орбитально устойчивыми однородным и противофазным решениями. При этом функиии $x_{1}=x_{*}(t, h, 2 l), x_{2}=x_{*}(t, h, 2 l)$ однородного режима содержат по $2 l$ промежутков положительности на периоде, а функиии $x_{1}=x_{*}(t, h-\Delta, l), x_{2}=x_{*}(t+\Delta, h-\Delta, l)$ противофазного решения содержат по l промежутков положительности. Здесъ

$$
\begin{aligned}
x_{*}(t, \theta, s) \stackrel{\text { def }}{=} \begin{cases}x_{0}(t), & t \in[0, \theta], \\
e_{k}(t, \theta, \theta), & t \in\left[\theta+k T_{0}, \theta+t_{0}+k T_{0}\right], k=0, \ldots, s-1, \\
t-\beta_{k}(\theta, \theta), & t \in\left[\theta+t_{0}+k T_{0}, \theta+(k+1) T_{0}\right], k=0, \ldots, s-1, \\
t-\beta_{s-1}(\theta, \theta), & t \in\left[\theta+s T_{0}, \beta_{s-1}(\theta, \theta)\right],\end{cases} \\
e_{k}(t, \theta, \tau) \stackrel{\text { def }}{=}\left(\alpha_{k}(\theta)-\frac{1}{b}-c\right) \exp \left(-b\left(t-\tau-k T_{0}\right)\right)+\frac{1}{b}+c, \\
\beta_{k}(\theta, \tau) \stackrel{\text { def }}{=} \tau+t_{0}+k T_{0}-\left(\alpha_{k}(\theta)-\frac{1}{b}-c\right) \xi-\frac{1}{b}-c, \\
\alpha_{k}(\theta) \stackrel{\text { def }}{=} \xi^{k}\left(x_{0}(\theta)-\eta\right)+\eta .
\end{aligned}
$$

Отдельно остановимся на вопросе, касающемся мультипликаторов импульсно-рефрактерного и синхронных режимов. В $[3,15,18]$ в процессе исследования устойчивости этих режимов доказаны следующие утверждения.

Лемма 2.1. Импульсно-рефрактерный режим из теоремы 2.1 имеет мультипликаторы $\mu_{1}=1, \mu_{2}=\xi+O\left(e^{-q \lambda}\right)(\lambda \rightarrow \infty)$, а все остальные его мультипликаторы $\mu_{j}(j=3,4, \ldots)$ экспоненииально маль.

Здесь и далее параметр $q$ - положительная константа, точное значение которой не важно.

Лемма 2.2. Однородный режим из теоремы 2.2 обладает единичным мультипликатором $\mu_{1}=1 ;$ мультипликатором

$$
\mu_{2}=2 \xi^{2 l}-1+O\left(e^{-q \lambda}\right), \quad \lambda \rightarrow \infty ;
$$

все остальные его мультипликаторы $\mu_{j}(j=3,4, \ldots)$ экспоненциально маль.

Лемма 2.3. Противофазный режсим из теоремы 2.2 обладает единичным мультипликатором $\mu_{1}=1 ;$ двумя мультипликаторами

$$
\mu_{2,3}=-\frac{1}{2}-\xi^{l} \pm i \frac{\sqrt{3-4 \xi^{l}}}{2}+O\left(e^{-q \lambda}\right), \quad \lambda \rightarrow \infty
$$

все остальные мультипликаторы $\mu_{j}(j=4,5, \ldots)$ экспоненииально малы. 
Отметим, что близость мультипликаторов (2.4), (2.5) к единичной окружности означает, что соответствующие им решения квазиустойчивы, т.е. траектория приближается к этому решению достаточно медленно, и в процессе вычислительного эксперимента выход на устойчивое решение может занять много времени.

3. Сосуществующие устойчивые циклы с несколькими всплесками на периоде. Перейдем к формулировке утверждений о сосуществовании у релейной задачи устойчивых циклов с различным числом областей положительности. Сперва дадим определение буферности.

Определение 3.1. Система обладает феноменом буферности, если по каждому натуральному числу $l$ можно так подобрать параметры, что система будет иметь $l$ устойчивых режимов с указанным механизмом их накопления.

Зафиксируем число $n \in \mathbb{N}$. Учитывая экспоненциальную зависимость величины $\xi$ от параметров $a$ и $b$, будем далее предполагать выполненными следующие неравенства:

$$
\begin{gathered}
a+\eta<0, \\
(n-1) T_{0}+\eta<0, \\
(n-1) T_{0}+t_{0}+1-\xi \eta \leqslant h \leqslant n T_{0}+\xi \eta .
\end{gathered}
$$

Сформулируем и докажем утверждение, означающее наличие буферности в системе (1.9).

Теорема 3.1. Пусть параметры $a, b, c, h$ удовлетворяют ограничениям (3.1)-(3.3). Тогда система (1.9) имеет $2 n-1$ периодических орбитально асимптотически устойчивых режимов, которые устроены следующим образом. Функиии $x_{1}$ и $x_{2}$ имеют соответственно $2 n-m$ и $(m=1, \ldots, 2 n-1)$ промежутков положительности, которые сменяются продолжительным промежутком отрицательности. Точные формулы решений имеют следующий вид:

$$
\begin{aligned}
& x_{1}^{(m)}(t) \stackrel{\text { def }}{=} \begin{cases}x_{0}(t), & t \in\left[0, h+d_{*}\right], \\
e_{k}\left(t, h+d_{*}, h+d_{*}\right), & t \in\left[h+d_{*}+k T_{0}, h+d_{*}+t_{0}+k T_{0}\right], \\
t-\beta_{k}\left(h+d_{*}, h+d_{*}\right), & k=0, \ldots, 2 n-m-1, \\
& t \in\left[h+d_{*}+t_{0}+k T_{0}, h+d_{*}+(k+1) T_{0}\right], \\
& k=0, \ldots, 2 n-m-1, \\
t-\beta_{2 n-m-1}\left(h+d_{*}, h+d_{*}\right), & t \in\left[h+d_{*}+(2 n-m) T_{0}, \beta_{2 n-m-1}\left(h+d_{*}, h+d_{*}\right)\right],\end{cases} \\
& \left(t-d_{*} \quad t \in\left[0, d_{*}\right]\right. \\
& x_{2}^{(m)}(t) \stackrel{\text { def }}{=} \begin{cases}x_{0}\left(t-d_{*}\right), & t \in\left[d_{*}, h\right], \\
e_{k}\left(t, h-d_{*}, h\right), & t \in\left[h+k T_{0}, h+t_{0}+k T_{0}\right], k=0, \ldots, m-1, \\
t-\beta_{k}\left(h-d_{*}, h\right), & t \in\left[h+t_{0}+k T_{0}, h+(k+1) T_{0}\right], k=0, \ldots, m-1, \\
t-\beta_{m-1}\left(h-d_{*}, h\right), & t \in\left[h+m T_{0}, \beta_{2 n-m-1}\left(h+d_{*}, h+d_{*}\right)\right],\end{cases} \\
& d_{*} \stackrel{\text { def }}{=} \frac{2(n-m) T_{0}+\xi^{m}\left(h-(2 n-m) T_{0}-\eta\right)-\xi^{2 n-m}\left(h-m T_{0}-\eta\right)}{2-\xi^{m}-\xi^{2 n-m}} .
\end{aligned}
$$

Здесь, как и раньше, $e_{k}$ и $\beta_{k}$ описываются формулами (2.1) и (2.2).

Возможный вид периодического режима проиллюстрирован на рис. 1.

Вследствие экспоненциальной связи (1.7) функций $u_{i}$ и $x_{i}, i=1,2$, свойства решения, описанные в теореме 3.1 , означают, что $u_{1}(t)$ и $u_{2}(t)$ обладают $m$ и $n-m$ всплесками соответственно. Это означает, что решению из теоремы 3.1 соответствует решение (1.1) c bursting-эффектом.

Доказательство теоремъ 3.1. Зафиксируем натуральное число $n$ и покажем наличие $2 n-1$ периодических режимов. Построим $n$ режимов, у которых количество промежутков положительности функции $x_{1}(t)$ на периоде не менее, чем у $x_{2}(t)$. Остальные $n-1$ режимов получим в силу 

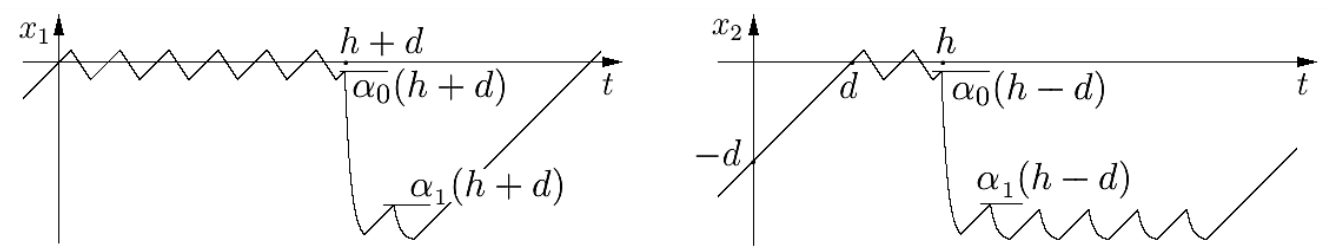

Рис. 1. Решение релейной системы (1.9). Здесь $n=4, m=2$

симметричного вхождения $x_{1}, x_{2}$ в (1.9). Для каждого режима необходимо определить множество начальных функций $S^{(m)}(m=1, \ldots, n)$, для которого будет построено отображение Пуанкаре, переводящее множество в себя. С этой целью зафиксируем положительную константу $\sigma$ :

$$
0<\sigma<\frac{a}{2},
$$

а также такие $q_{1}$ и $q_{2}$, что $q_{1} \in(0, \sigma), q_{2}>\sigma$, константы, зависящие от индекса $m(m=1, \ldots, n)$ :

$$
d_{1}^{(m)} \stackrel{\text { def }}{=}(n-m) T_{0}+\xi \eta, \quad d_{2}^{(m)} \stackrel{\text { def }}{=}(n-m) T_{0}-\xi \eta,
$$

и такие $q_{3}$ и $q_{4}$, что $q_{3} \in\left(0, d_{1}^{(m)}+\sigma\right), q_{4}>d_{2}^{(m)}+\sigma$. Определим два множества функций:

$$
\begin{gathered}
S_{1} \stackrel{\text { def }}{=}\left\{\varphi_{1} \in C[-h-\sigma,-\sigma]: \varphi_{1}(-\sigma)=-\sigma,-q_{2} \leqslant \varphi_{1}(t) \leqslant-q_{1} \forall t \in[-h-\sigma,-\sigma]\right\}, \\
S_{2}^{(m)} \stackrel{\text { def }}{=}\left\{\varphi_{2} \in C[-h-\sigma,-\sigma]: \varphi_{2}(-\sigma)=d-\sigma,-q_{4} \leqslant \varphi_{2}(t) \leqslant-q_{3} \forall t \in[-h-\sigma,-\sigma]\right\},
\end{gathered}
$$

где

$$
d \in\left[d_{1}^{(m)}, d_{2}^{(m)}\right], \quad m=1, \ldots, n .
$$

Определим набор множеств начальных функций системы (1.9) и значений параметра $d$ :

$$
S^{(m)} \stackrel{\text { def }}{=} S_{1} \times S_{2}^{(m)} \times\left[d_{1}^{(m)}, d_{2}^{(m)}\right], \quad m=1, \ldots, n .
$$

Отметим, что данное множество является замкнутым, ограниченным и выпуклым. Введем в рассмотрение оператор Пуанкаре. Для этого обозначим элемент множества $S^{(m)}$ через

$$
\varphi \stackrel{\text { def }}{=}\left(\varphi_{1}, \varphi_{2}, d\right), \quad \text { где } \quad \varphi_{1} \in S_{1}, \quad \varphi_{2} \in S_{2}^{(m)}, \quad d \in\left[d_{1}^{(m)}, d_{2}^{(m)}\right],
$$

а через

$$
x(t) \stackrel{\text { def }}{=}\left(x_{1}(t), x_{2}(t)\right)^{T}
$$

обозначим решение системы $(1.9)$, совпадающее на отрезке $[-h-\sigma,-\sigma]$ с начальной функцией $\varphi \in$ $S^{(m)}$ :

$$
x_{1}(t) \equiv \varphi_{1}(t), \quad x_{2}(t) \equiv \varphi_{2}(t) \quad \text { при } \quad t \in[-h-\sigma,-\sigma] .
$$

Предположим, что уравнение

$$
x_{1}(t-\sigma)=-\sigma
$$

имеет хотя бы $4 n-2 m$ положительных корней. Обозначим корень с номером $4 n-2 m$ через $T_{1}$. Зададим оператор последования Пуанкаре

$$
\Pi: C[-h-\sigma,-\sigma] \times C[-h-\sigma,-\sigma] \times\left[d_{1}^{(m)}, d_{2}^{(m)}\right] \rightarrow C[-h-\sigma,-\sigma] \times C[-h-\sigma,-\sigma] \times\left[d_{1}^{(m)}, d_{2}^{(m)}\right],
$$

определяя его равенством

$$
\Pi(\varphi) \stackrel{\text { def }}{=} x\left(t+T_{1}\right), \quad-h-\sigma \leqslant t \leqslant-\sigma .
$$

Найдем явную формулу, описывающую оператор (3.11). С этой целью отыщем решение системы (1.9) с начальной функцией из множества (3.9). 
Начнем с построения $x_{2}(t)$ на отрезке $t \in[-\sigma, d]$. Поскольку $d+\sigma<h$, при $t \in[-\sigma, d]$ выполняется $t-h<0$, т.е. $x_{2}(t-h)$ совпадает с отрицательной начальной функцией $\varphi_{2}(t) \in S_{2}$, а значит, $H\left(x_{2}(t-h)\right)=0$. Предположим, что неравенство

$$
x_{2}(t)<0
$$

сохраняется при $t \in(-\sigma, d-1]$; тогда при $t \in[-\sigma, d]$ выполняется $R\left(x_{2}(t-1)\right)=1$, а значит, для $x_{2}$ получаем здесь задачу Коши

$$
\dot{x}_{2}=1, \quad x_{2}(0)=-d .
$$

Отсюда получаем, что $x_{2}(t)=t-d$ при $t \in[0, d]$. Отметим, что априорное предположение (3.12) выполнено.

Далее, для функций $x_{1}, x_{2}$ на промежутках $t \in[0, h+d], t \in[d, h]$ соответственно имеем дело с уравнением

$$
\dot{x}_{j}=R\left(x_{j}(t-1)\right), \quad j=1,2 .
$$

При этом $x_{j}$ принимает нулевое значение в начальной точке рассматриваемого отрезка и начальные функции на интервале длины 1 отрицательны. Как было показано в [12], решение этой задачи совпадает с функцией $x_{0}(t)$. Таким образом, получаем, что

$$
x_{1}=x_{0}(t) \quad \text { при } t \in[0, h+d], \quad x_{2}=x_{0}(t-d) \quad \text { при } t \in[d, h] .
$$

Из условий (3.3), (3.8) следует справедливость неравенств

$$
(m-1) T_{0}+t_{0}+1 \leqslant h-d \leqslant m T_{0}, \quad(2 n-m-1) T_{0}+t_{0}+1 \leqslant h+d \leqslant(2 n-m) T_{0},
$$

которые означают, что точки $h+d$ и $h-d$ попадают на возрастающую фазу отрицательности периода функции $x_{0}$, причем $h+d$ попадает на $(2 n-m)$-й, а $h$ на $m$-й период.

Дальнейший анализ системы (1.9) связан с отрезками времени $\left[h+d, h+d+m T_{0}\right],[h, h+(2 n-$ $\left.m) T_{0}\right]$. Предполагаем a priori, что

$$
x_{1}(t)<0 \quad \text { при } h+d \leqslant t \leqslant h+d+m T_{0}, \quad x_{2}(t)<0 \quad \text { при } h \leqslant t \leqslant h+(2 n-m) T_{0} .
$$

Отметим, что на интервалах $(2 n-m-1) T_{0}+t_{0}<t<h+d, d+(m-1) T_{0}+t_{0}<t<h$ соответственно построенные функции $x_{1}(t), x_{2}(t)$ отрицательны, причем в силу условий $(3.14)$ длины этих интервалов больше единицы - первого запаздывания. Отсюда, с учетом (3.15), получаем, что $R\left(x_{j}(t-1)\right)=1, j=1,2$. Таким образом, решение здесь отыскивается из задач

$$
\begin{array}{ll}
\dot{x}_{1}=1+b\left(c-x_{1}\right) H\left(x_{0}(t-h-d)\right), & \left.x_{1}\right|_{t=h+d}=x_{0}(h+d), \\
\dot{x}_{2}=1+b\left(c-x_{2}\right) H\left(x_{0}(t-h)\right), & \left.x_{2}\right|_{t=h}=x_{0}(h-d) .
\end{array}
$$

Лемма 3.1. Решение задачи

$$
\dot{x}=1+b(c-x) H\left(x_{0}(t-\tau)\right),\left.\quad x\right|_{t=\tau}=x_{0}(\theta),
$$

описъвается формулой

$$
x(t)=\left\{\begin{array}{ll}
e_{k}(t, \theta, \tau), & t \in\left[\tau+k T_{0}, \tau+t_{0}+k T_{0}\right], \\
t-\beta_{k}(\theta, \tau), & t \in\left[\tau+t_{0}+k T_{0}, \tau+(k+1) T_{0}\right],
\end{array} \quad k \in\{0\} \cup \mathbb{N},\right.
$$

где $e_{k}(t, \theta, \tau), \beta_{k}(\theta, \tau)$ определень равенствами (2.1), (2.2).

Доказательство. Задача (3.18) распадается на последовательность задач Коши с начальными условиями, определяемыми предыдущим шагом. Обозначим последовательность начальных условий этих задач через $\left\{\alpha_{k}\right\}$ :

$$
\left.\alpha_{k} \stackrel{\text { def }}{=} x\right|_{t=\tau+k T_{0}}, \quad k \in\{0\} \cup \mathbb{N}
$$

в частности,

$$
\alpha_{0}=\alpha_{0}(\theta)=x_{0}(\theta)
$$


Покажем, что для $\alpha_{k}=\alpha_{k}(\theta)$ справедливо представление (2.3). На каждом из промежутков $t \in\left(\tau+k T_{0}, \tau+t_{0}+k T_{0}\right), k \in\{0\} \cup \mathbb{N}$, аргумент $x_{0}(t-\theta)$ функции $H$ в (3.18) положителен, а значит, здесь имеем дело с задачей

$$
\dot{x}=1+b(c-x),\left.\quad x\right|_{t=\tau+k T_{0}}=\alpha_{k}(\theta) .
$$

Решение данной задачи Коши описывается формулой

$$
x(t)=\left(\alpha_{k}(\theta)-\frac{1}{b}-c\right) \exp \left(-b\left(t-\tau-k T_{0}\right)\right)+\frac{1}{b}+c, \quad t \in b\left[\tau+k T_{0}, \tau+t_{0}+k T_{0}\right] .
$$

При $t \in\left(\tau+t_{0}+k T_{0}, \tau+(k+1) T_{0}\right), k \in\{0\} \cup \mathbb{N}$, функция $x_{0}(t-\tau)$ отрицательна, следовательно, $H\left(x_{0}(t-\tau)\right)=0$, и решение отыскивается из задачи

$$
\dot{x}=1,\left.\quad x\right|_{t=\tau+t_{0}+k T_{0}}=\left(\alpha_{k}(\theta)-\frac{1}{b}-c\right) \xi+\frac{1}{b}+c .
$$

В качестве решения задачи (3.23) получаем участок линейной функции:

$$
x(t)=t-\tau-t_{0}-k T_{0}+\left(\alpha_{k}(\theta)-\frac{1}{b}-c\right) \xi+\frac{1}{b}+c, \quad t \in\left[\tau+t_{0}+k T_{0}, \tau+(k+1) T_{0}\right] .
$$

Из формул (3.24), (1.5) следует, что последовательность чисел $\left\{\alpha_{k}(\theta)\right\}$ задается рекуррентным соотношением

$$
\alpha_{k+1}(\theta)=\left(\alpha_{k}(\theta)-\frac{1}{b}-c\right) \xi+\frac{1}{b}+c+a+1
$$

откуда, с учетом (3.21), вытекает явная формула (2.3).

Суммируя вышесказанное, получаем, что решением задачи (3.18) является функция (3.19). Лемма 3.1 доказана.

Вернемся к рассмотрению задач (3.16), (3.17). Из (3.19) следует, что для $x_{1}(t), x_{2}(t)$ соответственно на отрезках $t \in\left[h+d, h+d+m T_{0}\right], t \in\left[h, h+(2 n-m) T_{0}\right]$ справедливы следующие формулы:

$$
\begin{aligned}
& x_{1}(t)= \begin{cases}e_{k}(t, h+d, h+d), & t \in\left[h+d+k T_{0}, h+d+t_{0}+k T_{0}\right], \\
t-\beta_{k}(h+d, h+d), & t \in\left[h+d+t_{0}+k T_{0}, h+d+(k+1) T_{0}\right],\end{cases} \\
& x_{2}(t)=\left\{\begin{array}{ll}
e_{k}(t, h-d, h), & t \in\left[h+k T_{0}, h+t_{0}+k T_{0}\right], \\
t-\beta_{k}(h-d, h), & t \in\left[h+t_{0}+k T_{0}, h+(k+1) T_{0}\right],
\end{array} \quad k=1, \ldots, 2 n-m .25\right)
\end{aligned}
$$

Из формул $(1.6),(3.21)$ получаем $\alpha_{0}(h+d)=h+d-(2 n-m) T_{0}, \alpha_{0}(h-d)=h-d-m T_{0}$, откуда следует, что

$$
-a<\alpha_{0}(h+d)<0, \quad-a<\alpha_{0}(h-d)<0 .
$$

Отметим, что последовательности $\left\{\alpha_{k}(h-d)\right\},\left\{\alpha_{k}(h+d)\right\}$ убывают, поскольку коэффициент $\left(x_{0}(\theta)-\eta\right)$ в формуле (3.20) положителен в силу условий $(3.1),(3.27)$, а число $\xi<1-$ положительная константа.

Тогда, с учетом убывания, все элементы последовательности $\left\{\alpha_{k}(\theta)\right\}$ отрицательны, что обеспечивает достаточно «длинный» промежуток отрицательности функций $x_{1}(t), x_{2}(t)$. Это означает, что наше априорное предположение (3.15) справедливо. Таким образом, последовательность $\left\{\alpha_{k}(\theta)\right\}$ при $\theta=h+d$ и $\theta=h-d$ - это значения функций $x_{1}(t), x_{2}(t)$ в отрицательной части (см. рис. 1). Также отсюда следует, что при $h+d+m T_{0}<t \leqslant h+d+m T_{0}+1$ имеем $x_{1}(t-1)<0$, $x_{1}(t-h)<0$. Аналогично, при $h+(2 n-m) T_{0}<t \leqslant h+(2 n-m) T_{0}+1$ имеем $x_{2}(t-1)<0$, $x_{2}(t-h)<0$. Поэтому функции $x_{1}(t), x_{2}(t)$ здесь определяются из задач Коши

$$
\begin{aligned}
& \dot{x}_{1}=1,\left.\quad x_{1}\right|_{t=h+d+m T_{0}}=\alpha_{m}(h+d), \\
& \dot{x}_{2}=1,\left.\quad x_{2}\right|_{t=h+(2 n-m) T_{0}}=\alpha_{2 n-m}(h-d),
\end{aligned}
$$


следовательно, задаются равенствами

$$
\begin{aligned}
& x_{1}(t)=t+\alpha_{m}(h+d)-h-d-m T_{0}, \\
& x_{2}(t)=t+\alpha_{2 n-m}(h-d)-h-(2 n-m) T_{0} .
\end{aligned}
$$

Добавим еще, что формула (3.30) сохраняется при тех $t>h+d+m T_{0}$, для которых одновременно $x_{1}(t-1)<0$ и $x_{1}(t-h)<0$. Тем самым, она заведомо применима при $h+d+m T_{0} \leqslant t<$ $-\alpha_{m}(h+d)+h+d+m T_{0}+1$. По аналогичным соображениям формула (3.31) распространяется на промежуток $h+(2-m) T_{0} \leqslant t<-\alpha_{2 n-m}(h-d)+h+(2 n-m) T_{0}+1$.

Итак, найдены явные формулы для оператора (3.11). Распорядимся теперь выбором свободного параметра $\sigma$ из (3.9). В дальнейшем будем считать, что наряду с (3.7) выполнено условие

$$
\sigma<\min \left\{\alpha_{0}(h-d), \alpha_{0}(h+d)\right\}
$$

с номером $2 m+1$. Из полученных равенств $(3.13),(3.25),(3.26),(3.30)$ и (3.32) следует, что

$$
\begin{aligned}
& T_{1}=h+d+m T_{0}-\alpha_{m}(h+d), \\
& T_{2}=h+(2 n-m) T_{0}-\alpha_{2 n-m}(h-d) .
\end{aligned}
$$

Отметим, что в силу неравенств $(3.2),(3.32)$ длина отрезка отрицательности между $(4 n-2 m-$ $1)$-м и $(4 n-2 m)$-м корнями уравнения $(3.10)$ (которые равны $(2 n-m-1) T_{0}+t_{0}+\sigma t_{0}$ и $\left.T_{1}\right)$ больше длины $h$ отрезка, на котором определено множество $S^{(m)}$. Это означает, что построенный оператор переводит компактное множество $S^{(m)}$ в себя, откуда следует, что для каждого $m=1, \ldots, n$ существует периодическое решение релейной системы, описанное в теореме 3.1 . Из явных формул для оператора П становится ясно, что он является сжимающим, поскольку всем функциям из множества $S^{(m)}$ ставится в соответствие одна функция, описанная формулами (3.4), (3.5). Таким образом, в соответствии с принципом сжимающих отображений оператор П обладает единственной неподвижной точкой в $S^{(m)}$; следовательно, соответствующее периодическое решение системы $(1.9)$ с начальной функцией из $S^{(m)}$ является единственным. Кроме того, сжимающие свойства П означают, что в спектре устойчивости найденного периодического режима кроме единичного мультипликатора есть еще один мультипликатор $\mu \neq 0$, а все остальные мультипликаторы равны нулю (их счетное число в силу бесконечномерности оператора П). Мультипликатор $\mu$ при этом является мультипликатором отщепляющегося от П одномерного отображения, сопоставляющего числу $-d$ величину $-\tilde{d} \stackrel{\text { def }}{=} x_{2}\left(T_{1}\right)$. Отыщем значение $\tilde{d}$. В силу ограничений (3.2) величина $T_{1}$ попадает на «длинный» участок $\left[h+d+m T_{0}, T_{2}\right]$ линейного возрастания функции $x_{2}(t)$, где справедлива формула $x_{2}(t)=t-T_{2}$. Отсюда

$$
\begin{aligned}
& \tilde{d}=T_{2}-T_{1}=h+(2 n-m) T_{0}-\alpha_{2 n-m}(h-d)-h-d-m T_{0}+\alpha_{m}(h+d)= \\
&=-d+(2 n-2 m) T_{0}-\xi^{2 n-m}\left(h-d-m T_{0}-\eta\right)+\xi^{m}\left(h+d-(2 n-m) T_{0}-\eta\right)= \\
&=\left(-1+\xi^{m}+\xi^{2 n-m}\right) d+2(n-m) T_{0}-\xi^{2 n-m}\left(h-m T_{0}-\eta\right)+\xi^{m}\left(h-(2 n-m) T_{0}-\eta\right) .
\end{aligned}
$$

Неподвижная точка данного отображения - величина (3.6). Из (3.35) следует, что $\mu=-1+$ $\xi^{m}+\xi^{2 n-m}$. Таким образом, относительно мультипликаторов найденного периодического решения релейной системы (1.9) справедливо следующее утверждение.

Лемма 3.2. Режимы из теоремы 3.1 обладают единичным мультипликатором $\mu_{1}=1$; мультипликатором

$$
\mu_{2}=-1+\xi^{m}+\xi^{2 n-m}+O\left(e^{-q \lambda}\right), \quad \lambda \rightarrow \infty
$$

все остальные мультипликаторь $\mu_{j}(j=3,4, \ldots)$ экспоненицально маль.

Отметим, что так же, как в леммах 2.2, 2.3, в данном случае получаем мультипликатор, по модулю близкий к единице. Таким образом, режимы релейной системы (1.9), описанные в теореме 3.1, оказываются квазиустойчивыми. 
4. Численное моделирование. В пп. 2 и 3 при значениях $\lambda \rightarrow \infty$ показаны существование и устойчивость режимов, у которых один или несколько мультипликаторов близки по модулю к единице, причем эта близость не является асимптотической, а обусловлена малостью величины $\xi^{n}$, где, напомним, $\xi=\exp \left(-b t_{0}\right)$. При обычно (см. [5]) рассматриваемых значениях параметров $a=1,5, b=2\left(t_{0}=1+1 / a\right.$ в этом случае равно $\left.5 / 3\right)$ величина $\xi$ оказывается равной $\exp (-10 / 3)$, что при $n$, например, равном 8 приводит к $\xi^{8} \approx 2 \cdot 10^{-12}$. Это означает, что решения системы (1.1), находящиеся в бассейне притяжения одного из рассматриваемых в пп. 2 и 3 циклов, на промежутке длины периода устойчивого решения претерпевают изменения, по воздействию близкие к $1-\xi^{n}$, что обуславливает очень длинный переходный процесс, который, как нетрудно видеть, реализуется и при значениях $\lambda$, не являющихся асимптотически большими. Таким образом, численный анализ задачи (1.1) сопряжен с существенными трудностями. В случае, если параметр $\lambda$ не велик, эти трудности могут остаться незамеченными, и тогда в качестве устойчивого режима, полученного на основе вычислительного эксперимента, может быть предъявлена часть переходного процесса, что приведет к неверным результатам. Для того чтобы продиагностировать наличие такого явления, нами предлагается следующая последовательность действий.

На первом этапе выполняется вычисление решения задачи (1.1) со специальным образом подобранными начальными условиями до тех пор, пока решение не выйдет на участок медленного приближения к некоторому возможно периодическому решению. Затем, на втором этапе, выполняется линеаризация вдоль рассматриваемого решения системы (1.1) и вычисляются показатели Ляпунова для этого решения.

Отметим, что ляпуновские показатели для систем с запаздыванием могут не вполне корректно оцениваться численно. Дело в том, что для конечномерных систем имеет место известная теорема Оселедеца (см. [13]), в которой утверждается, что линеаризованная на устойчивом решении система является правильной по Ляпунову. Это позволяет в определении ляпуновских показателей (см. [2]) заменить верхний предел на обычный и численно оценивать эти величины. В случае уравнения с запаздыванием такого утверждения не доказано, однако в целом ряде численных экспериментов (см. [1]) демонстрируется «близость» оцениваемых инвариантных характеристик к показателям Ляпунова.

В настоящей работе применяется расширение стандартного алгоритма (см. [22]) для вычисления нескольких первых показателей Ляпунова для систем дифференциальных уравнений с запаздывающими аргументами. Так как изучаемые решения системы (1.1) при большом $\lambda$ содержат интенсивные высокоамплитудные всплески, которые перемежаются участками, где решение близко к нулю, для удобства численно будем изучать систему после экспоненциальной замены (1.8).

Детально опишем алгоритм получения первых $K$ показателей Ляпунова на некотором решении системы (1.8); при этом в качестве функций $f(u)$ и $g(u)$, удовлетворяющих условиям (1.3) и (1.4), выберем функции следующего вида: $f(u)=(a-a u)(a+u)^{-1}, g(u)=u(1+u)^{-1}$. В качестве численного метода для решения системы (1.8) с начальными условиями

$$
x_{1}(t)=\psi_{1}(t), \quad x_{2}(t)=\psi_{2}(t), \quad t \in[-h, 0], \quad\left(\psi_{1}(t), \psi_{2}(t)\right)^{T} \in C\left([-h, 0] ; \mathbb{R}^{2}\right)
$$

будем использовать метод Дормана-Принса пятого порядка (DOPRI54) с переменной длиной шага интегрирования (см. [17]).

Таким образом, будем решать систему (1.8) с начальным условием (4.1) выбранным методом до момента времени $\Theta$, достаточного для приближения траектории решения к изучаемому аттрактору. При этом на промежутке $t \in[\Theta-h, \Theta]$ получим функцию $x^{(0)}(t) \in C\left([\Theta-h, \Theta] ; \mathbb{R}^{2}\right)$, которая станет новым начальным условием системы (1.8).

Далее, дополним систему уравнений (1.8) следующими $K$ идентичными системами:

$$
\begin{aligned}
\dot{v}_{1 j}=-\lambda a v_{1 j}(t-1) \frac{(1+a) \exp \left(\lambda x_{1}^{*}(t-1)\right)}{\left(a+\exp \left(\lambda x_{1}^{*}(t-1)\right)\right)^{2}}-b v_{1 j} \frac{\exp \left(\lambda x_{2}^{*}(t-h)\right)}{1+\exp \left(\lambda x_{2}^{*}(t-h)\right)} & \begin{aligned}
& +\lambda b\left(c-x_{1}^{*}\right) \frac{\exp \left(\lambda x_{2}^{*}(t-h)\right)}{\left(1+\exp \left(\lambda x_{2}^{*}(t-h)\right)\right)^{2}} v_{2 j}(t-h)
\end{aligned}
\end{aligned}
$$



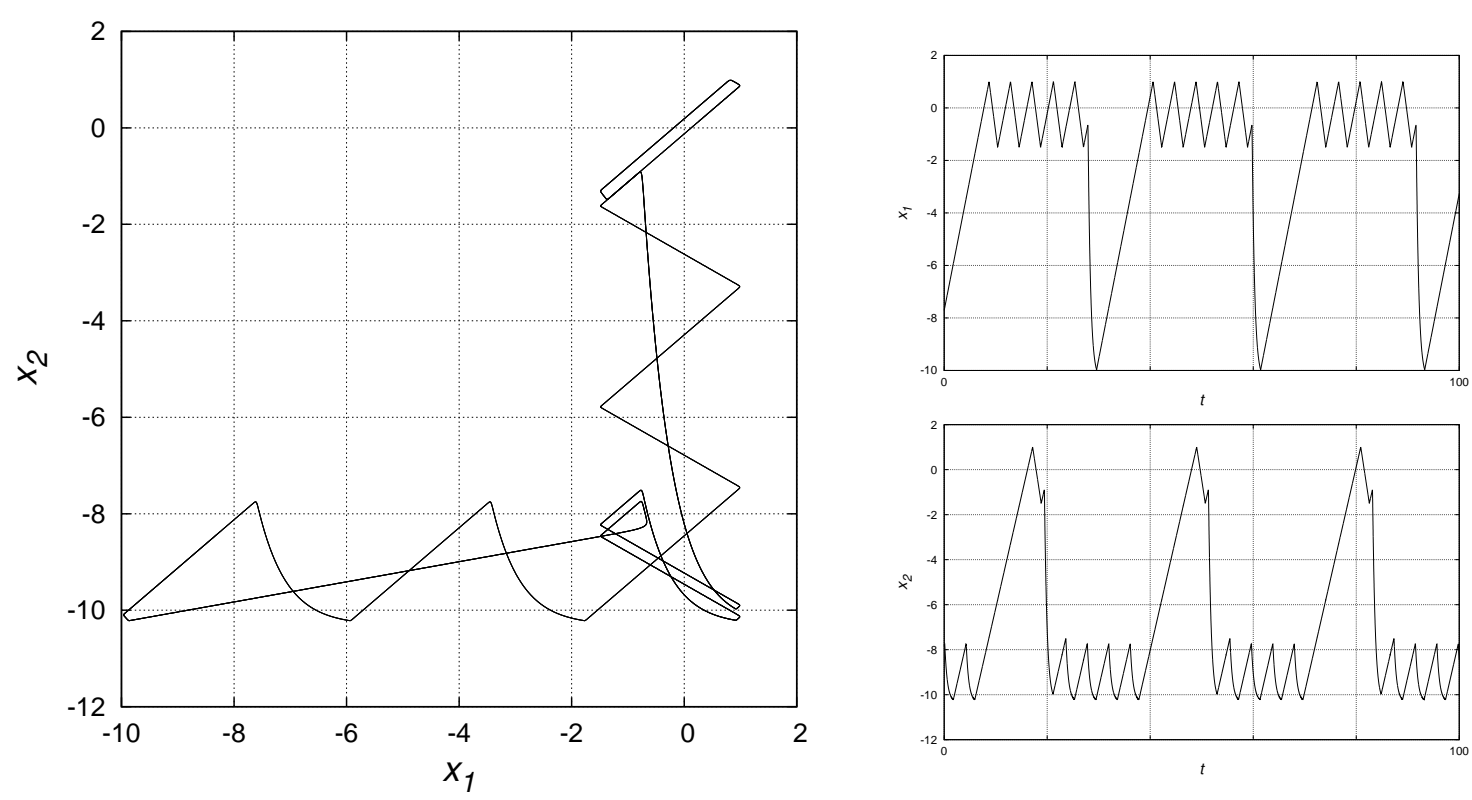

Рис. 2. Периодическое решение с пятью промежутками положительности первой компоненты и одним второй компоненты (а)
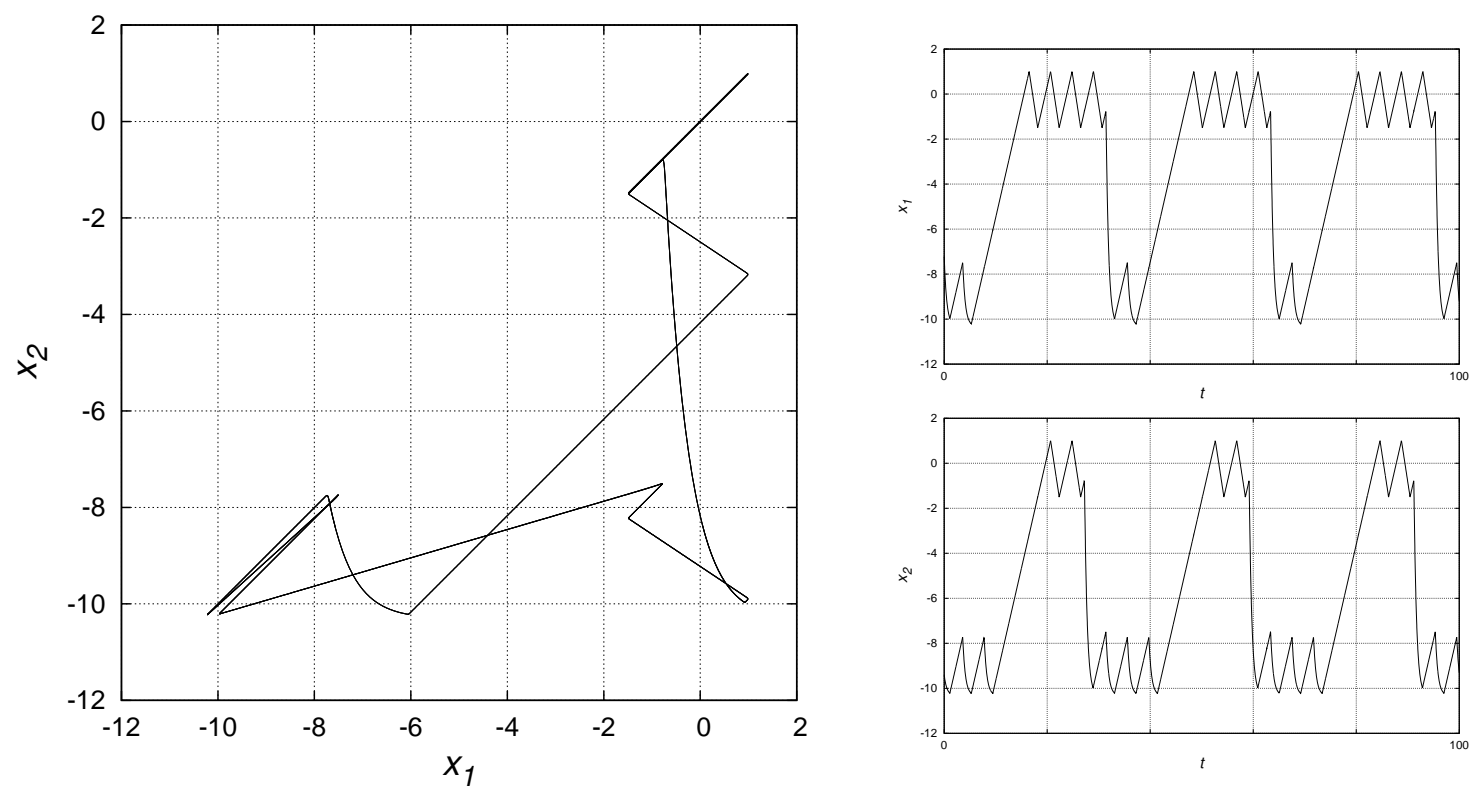

Рис. 3. Периодическое решение с четырьмя промежутками положительности первой компоненты и двумя второй компоненты (b)

$$
\begin{aligned}
\dot{v}_{2 j}=-\lambda a v_{2 j}(t-1) \frac{(1+a) \exp \left(\lambda x_{2}^{*}(t-1)\right)}{\left(a+\exp \left(\lambda x_{2}^{*}(t-1)\right)\right)^{2}} & -b v_{2 j} \frac{\exp \left(\lambda x_{1}^{*}(t-h)\right)}{1+\exp \left(\lambda x_{1}^{*}(t-h)\right)} \\
& +\lambda b\left(c-x_{2}^{*}\right) \frac{\exp \left(\lambda x_{1}^{*}(t-h)\right)}{\left(1+\exp \left(\lambda x_{1}^{*}(t-h)\right)\right)^{2}} v_{1 j}(t-h),
\end{aligned}
$$



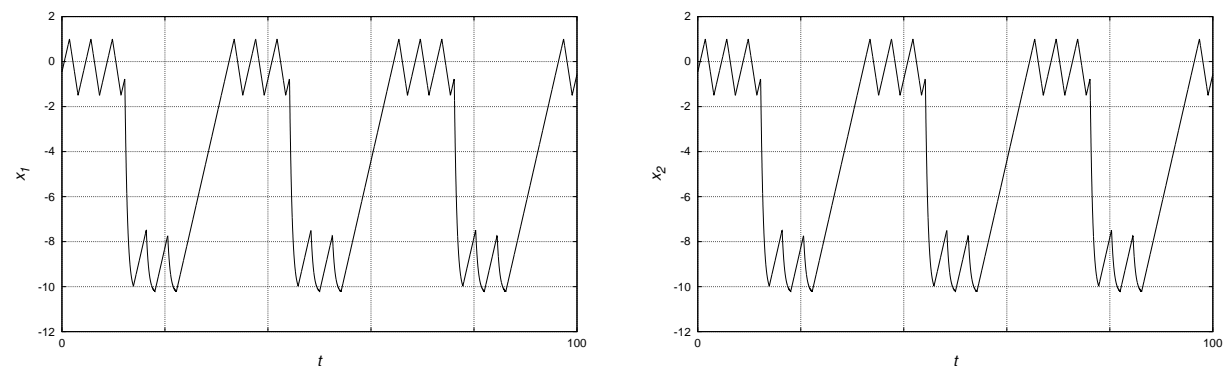

Рис. 4. Однородный режим с суммарно шестью промежутками положительности (с)
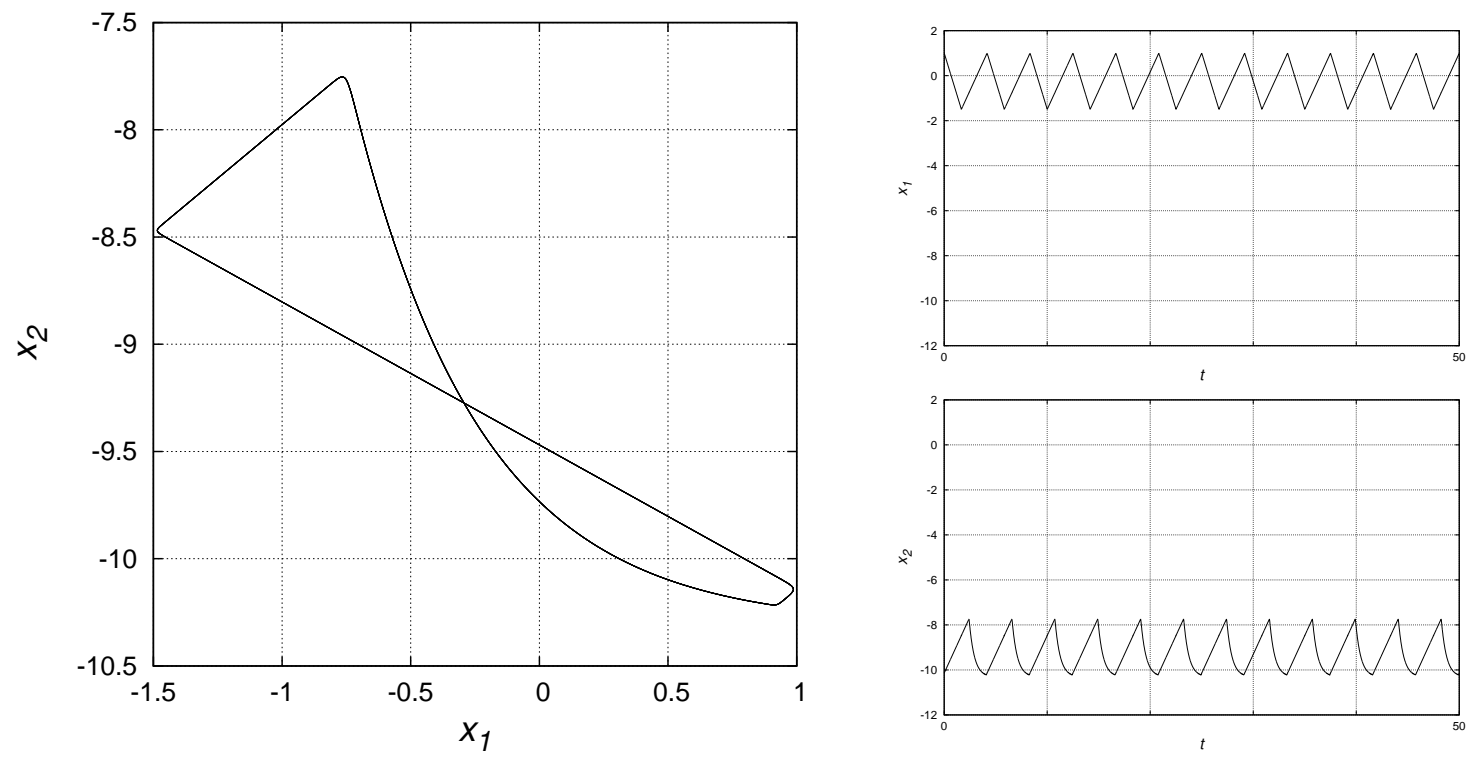

Рис. 5. Импульсно-рефрактерный режим (d)

где $j=1, \ldots, K, x^{*}(t)=\left(x_{1}^{*}(t), x_{2}^{*}(t)\right)^{T}$ - решение системы уравнений (1.8) с начальным условием $x(t)=x^{(0)}(t)$ при $t>\Theta$. Они представляют собой линеаризованные на решении $x^{*}(t)$ системы уравнений (1.8). Для каждой системы уравнений (4.2) используем начальные условия в виде ортонормированных импульсных функций, например:

$$
v_{1 j}(t)=v_{2 j}(t)=\left\{\begin{array}{cl}
\sqrt{\frac{K}{2 h}} & \text { при } t \in\left[(\Theta-h)+(j-1) \frac{h}{K},(\Theta-h)+j \frac{h}{K}\right], \quad j=1, \ldots, K, \\
0 & \text { иначе. }
\end{array}\right.
$$

Далее будем использовать обозначение $v_{j}(t) \stackrel{\text { def }}{=}\left(v_{1 j}(t), v_{2 j}(t)\right)^{T}, j=1, \ldots, K$; введем норму

$$
\left\|v_{j}(t)\right\|^{2} \stackrel{\text { def }}{=} \int_{t-h}^{t}\left|v_{1 j}(\tau)\right|^{2} d \tau+\int_{t-h}^{t}\left|v_{2 j}(\tau)\right|^{2} d \tau
$$

Решая совместно систему (1.8) с начальным условием $x(t)=x^{(0)}(t)$ и системы уравнений $(4.2)$ с начальными условиями (4.3) на промежутке $t \in\left[\Theta, \Theta+T^{*}\right], T^{*} \geqslant h$, получаем для каждой из линеаризованных систем решение $v_{j}^{(1)}(t)=\left(v_{1 j}^{(1)}(t), v_{2 j}^{(1)}(t)\right)^{T}, j=1, \ldots, K$. 

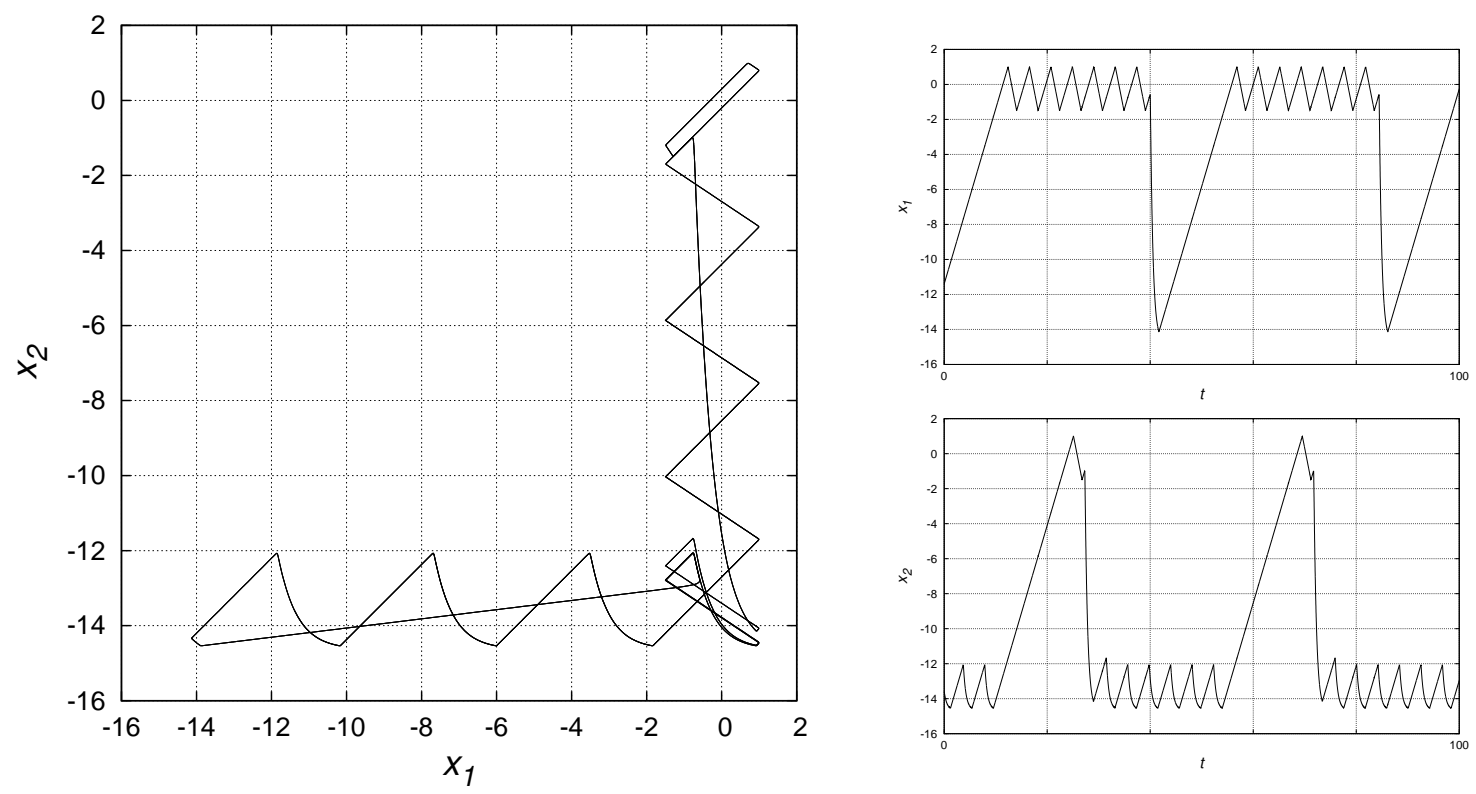

Рис. 6. Периодическое решение с семью промежутками положительности первой компоненты и одним второй компоненты (е)

Учитывая, что решения $v_{j}^{(1)}(t)$, вообще говоря, ведут себя экспоненциально, необходимо их перенормировывать через определенные промежутки времени. Отметим, что проблему представляют как неограниченный рост решений, так и их стремление к нулю. Таким образом, на промежутке $t \in\left[\Theta+T^{*}-h, \Theta+T^{*}\right]$ разбиваем полученные решения линеаризованных систем на $M$ равных временных интервалов, усредняя внутри каждого интервала соответствующие ему значения $v_{1 j}^{(1)}(t)$ и $v_{2 j}^{(1)}(t)$, в результате чего получаем кусочно непрерывные функции $\tilde{v}_{1 j}^{(1)}(t)$ и $\tilde{v}_{2 j}^{(1)}(t)$ соответственно, которые используем в методе импульсных функций:

(i) применяем метод Грама-Шмидта (см. [16]) к $\tilde{v}_{j}^{(1)}(t)$;

(ii) после ортогонализации, но перед перенормировкой вычисляем величины $\nu_{j}^{(1)} \stackrel{\text { def }}{=}\left\|\tilde{v}_{\text {ort } j}^{(1)}\right\|$, где $\tilde{v}_{\text {ort } j}^{(1)}(t)$ - ортогонализированная система функций $\tilde{v}_{j}^{(1)}(t), j=1, \ldots, K$;

(iii) продолжаем решать системы (1.8), (4.2), при этом в качестве начальных условий для линеаризованных систем используем полученную ортонормированную систему функций.

Повторяем описанный процесс на временных интервалах $\left[\Theta+k T^{*}-h, \Theta+k T^{*}\right], k>1$, в результате чего обработке методом импульсных функций подвергаются соответствующие решения $v_{j}^{(k)}(t)$. Оценка показателей Ляпунова в таком случае вычисляется по формуле

$$
\lambda_{j}=\lim _{L \rightarrow \infty} \frac{1}{L T^{*}} \sum_{k=1}^{L} \ln \nu_{j}^{(k)}, \quad j=1, \ldots, K .
$$

Теперь перейдем к результатам численного исследования системы (1.8). Во всех экспериментах применялись следующие параметры:

(a) количество вычисляемых показателей Ляпунова $K=4$;

(b) время $\Theta$ выхода на аттрактор - от 1000 до 3000, при этом в зависимости от скорости сходимости решения к моделируемому режиму не учитывалось в оценке (4.4) некоторое количество вычисленных норм из первых шагов обработки методом импульсных функций;

(c) интервал до следующей перенормировки решений линеаризованных систем $T^{*}=h$;

(d) количество $M$ временных интервалов разбиения решений линеаризованных систем -1000 ;

(e) параметр $\lambda=100$. 

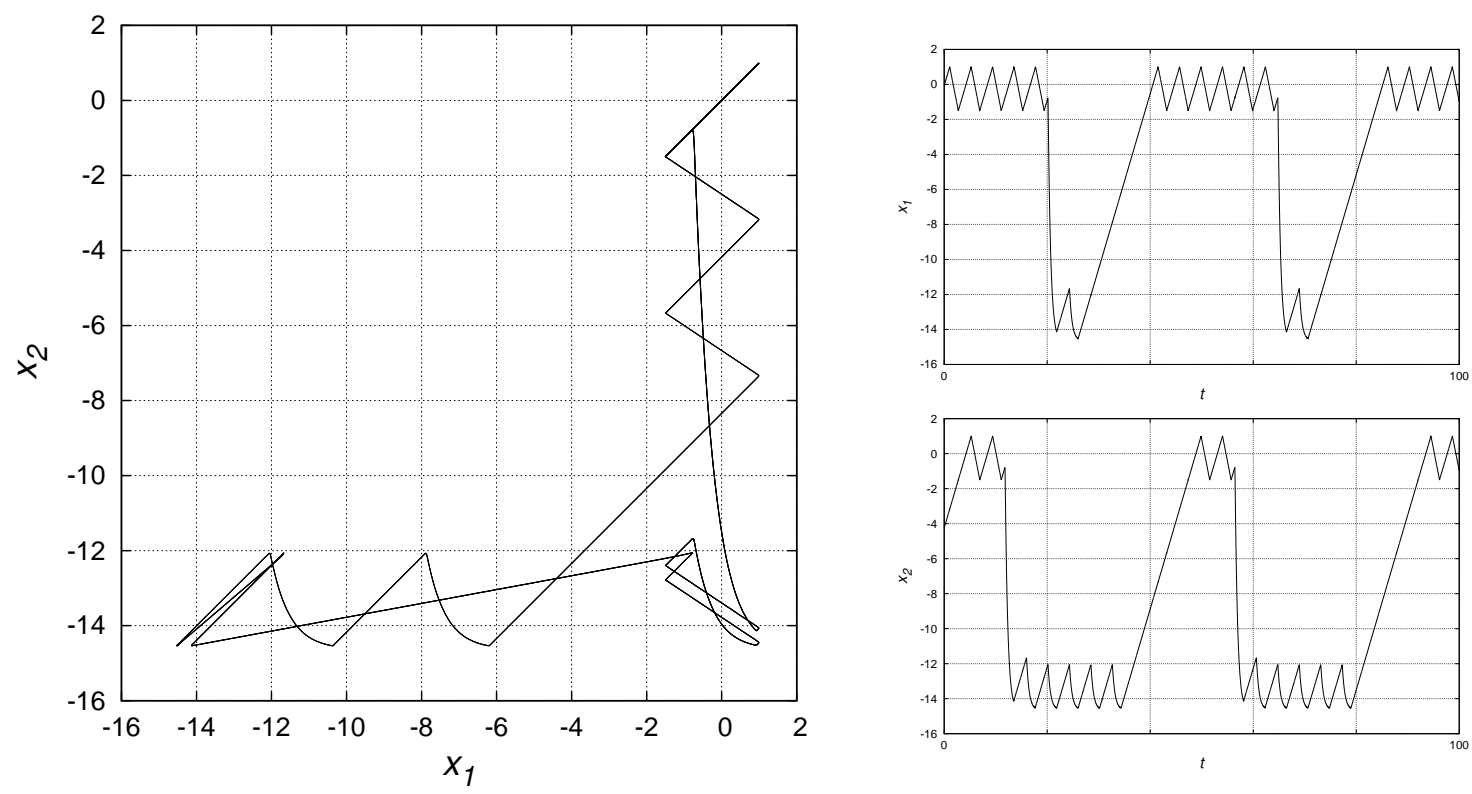

Рис. 7. Периодическое решение с шестью промежутками положительности первой компоненты и двумя второй компоненты (f)
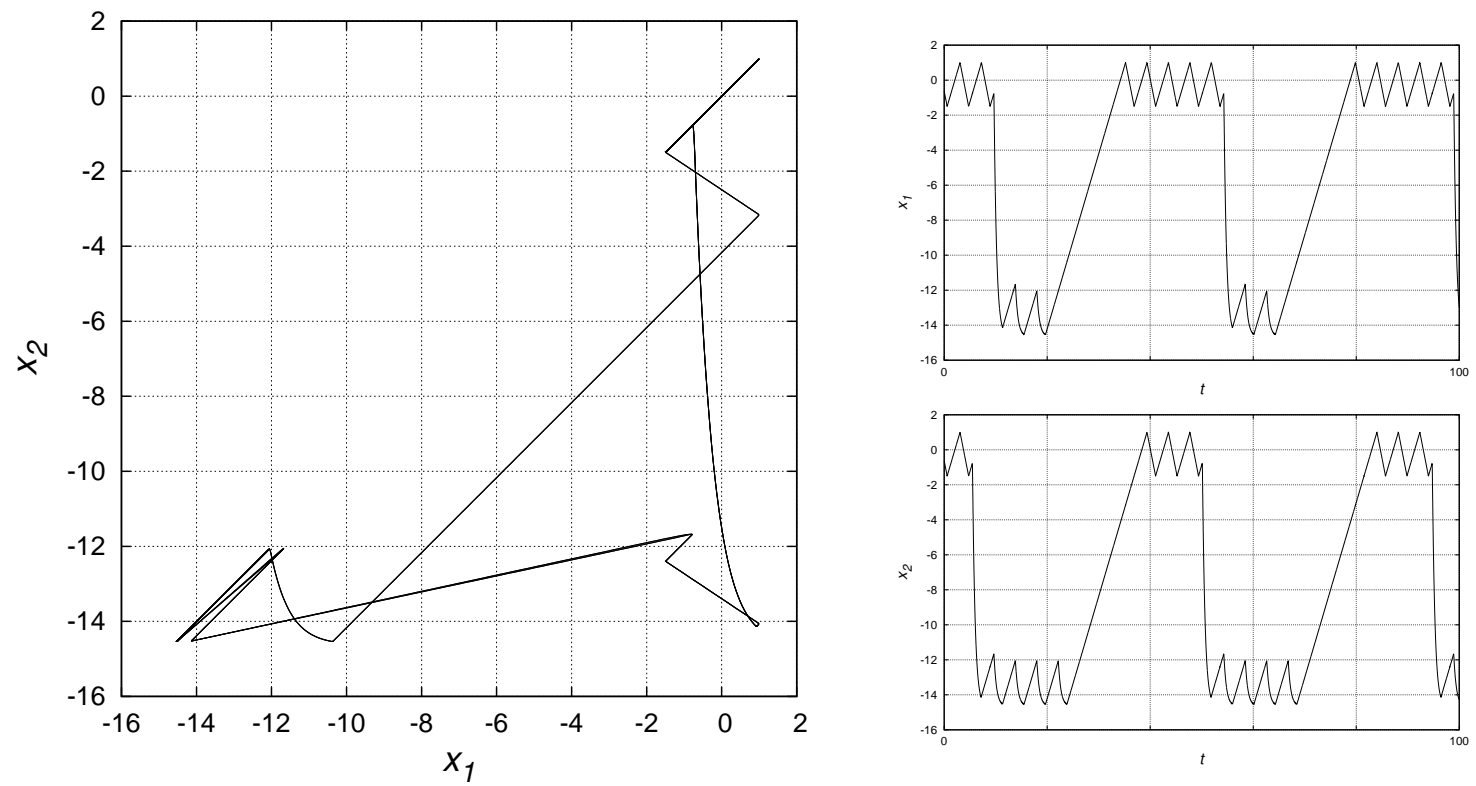

Рис. 8. Периодическое решение с пятью промежутками положительности первой компоненты и тремя второй компоненты (g)

Значения показателей Ляпунова для удобства округлены до четвертого знака после запятой.

Далее представлены комплекты сосуществующих режимов изучаемой в предыдущих пунктах структуры при разных значениях параметров $a, b, c$ и $h$. Наличие у моделируемых режимов нескольких промежутков положительности на периоде означает, что соответствующие режимы 

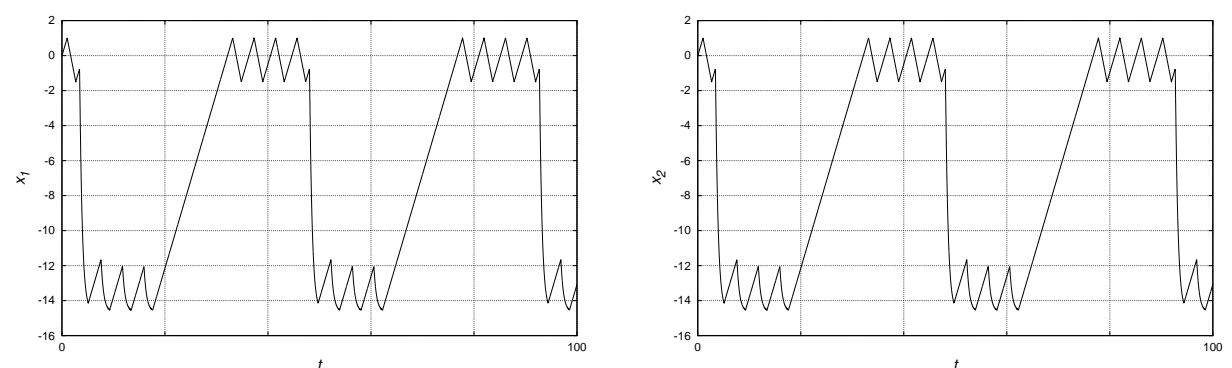

Рис. 9. Однородный режим с суммарно восемью промежутками положительности (h)
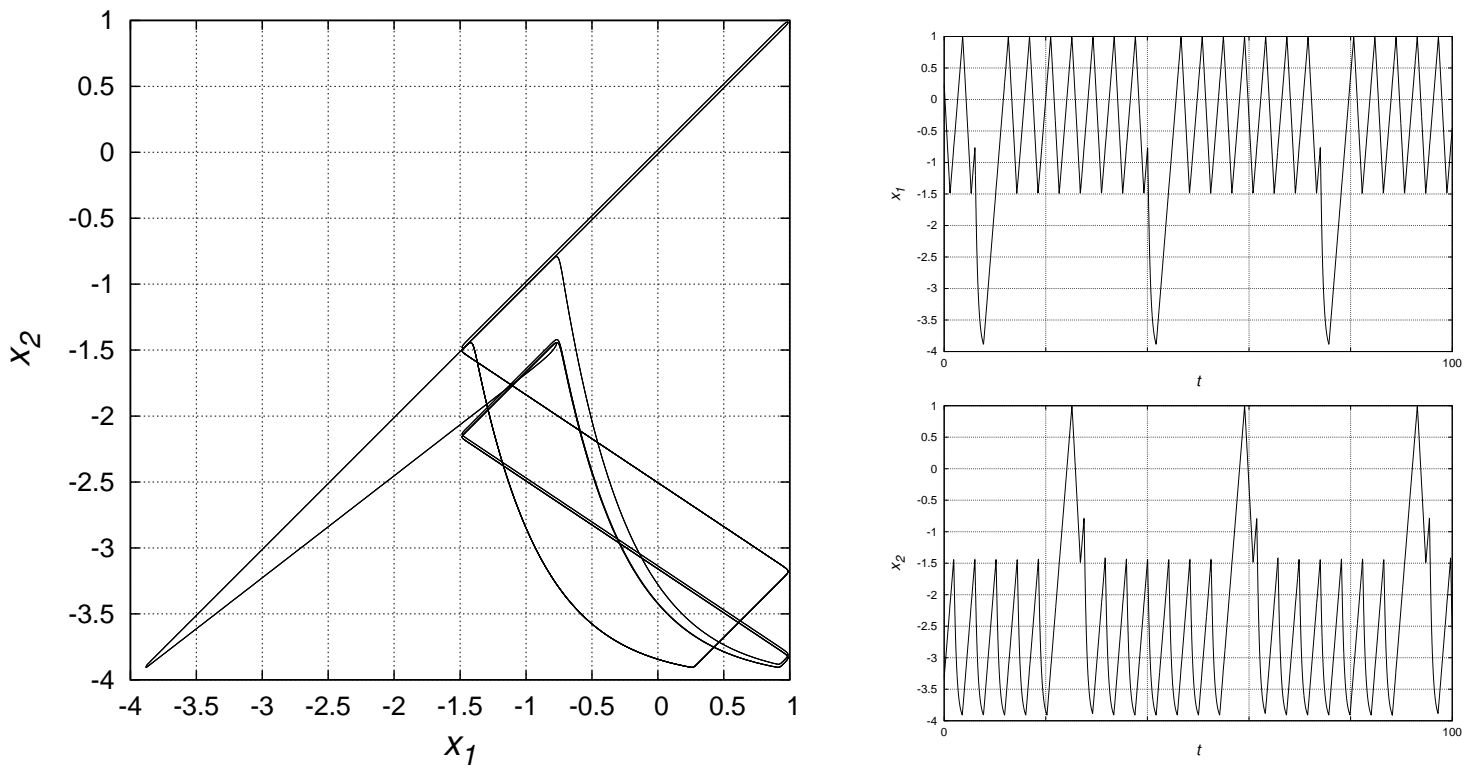

Рис. 10. Периодическое решение с семью промежутками положительности первой компоненты и одним второй компоненты (i)

Таблица 1. Первые четыре показателя Ляпунова сосуществующих режимов (a)-(d) при $a=1.5, b=2, c=-10.81, h=11.75$

\begin{tabular}{|c|c|r|c|c|}
\hline & $\lambda_{1}$ & \multicolumn{1}{c|}{$\lambda_{2}$} & \multicolumn{1}{c|}{$\lambda_{3}$} & $\lambda_{4}$ \\
\hline (a) & 0,0000 & $-0,0011$ & $-1,2020$ & $-1,2050$ \\
(b) & 0,0000 & 0,0000 & $-1,1966$ & $-1,2070$ \\
(c) & 0,0000 & 0,0000 & $-1,1918$ & $-1,2084$ \\
(d) & 0,0000 & $-0,8033$ & $-3,3004$ & $-3,6840$ \\
\hline
\end{tabular}

исходной модели (1.1) обладают bursting-эффектом. Отметим, что импульсно-рефрактерный режим удается получить при всех представленных наборах параметров, поскольку они удовлетворяют условию теоремы 2.1, но для краткости такой режим будет проиллюстрирован лишь в случае первого набора из-за однотипного графического представления и сходства устройства спектров показателей Ляпунова. 

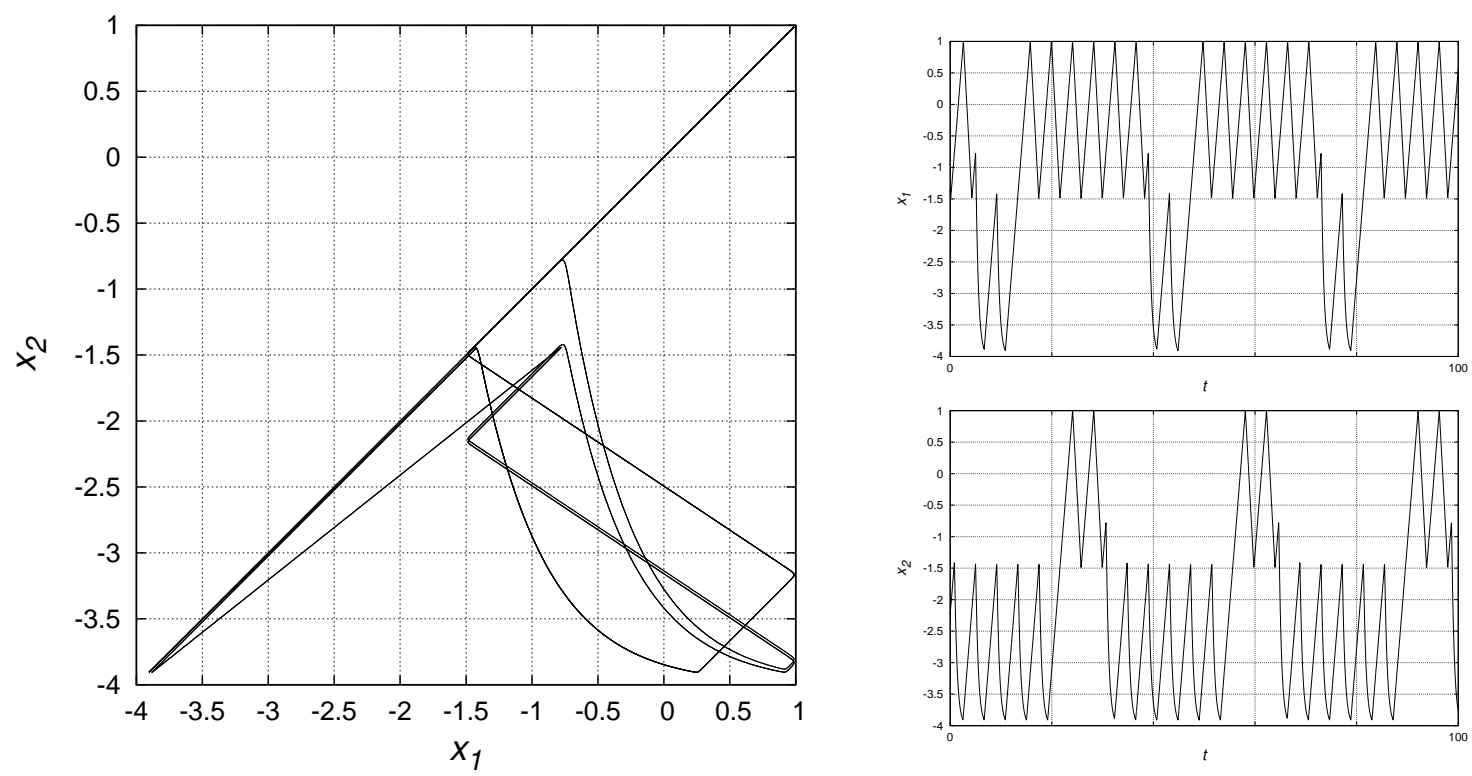

Рис. 11. Периодическое решение с шестью промежутками положительности первой компоненты и двумя второй компоненты $(\mathrm{j})$
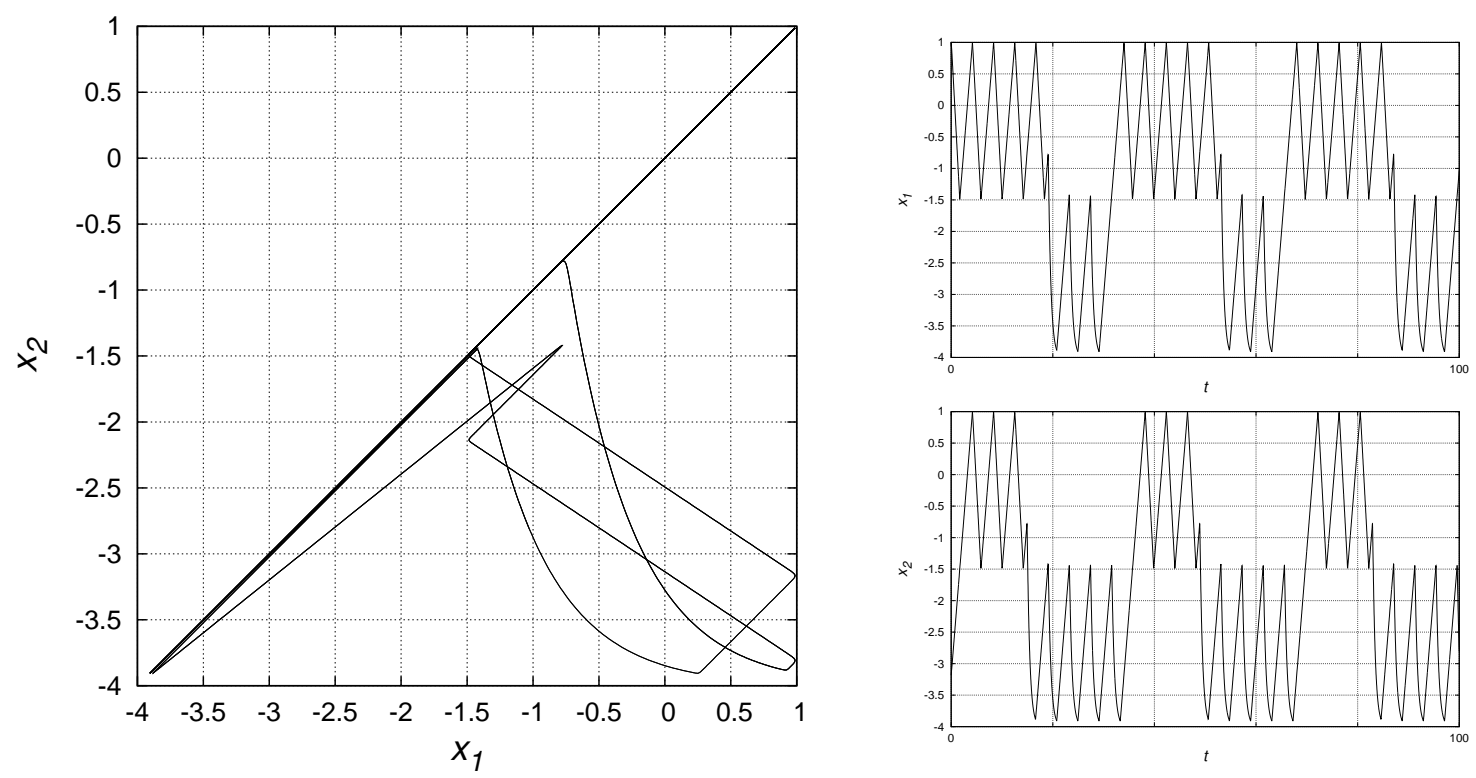

Рис. 12. Периодическое решение с пятью промежутками положительности первой компоненты и тремя второй компоненты $(\mathrm{k})$

Сначала рассмотрим режимы при $a=1,5, b=2, c=-10,81, h=11,75$ (см. рис. $2-5$ и таблицу 1). Режимы (a)-(c) обладают суммарно шестью промежутками положительности на длине периода. Режим (c) является однородным, поэтому для этого режима (и подобных однородных режимов при других параметрах) представлены лишь графики изменения компонент системы по времени. Близость к нулю вторых показателей Ляпунова у режимов (a), (b) и (c) подтверждает 

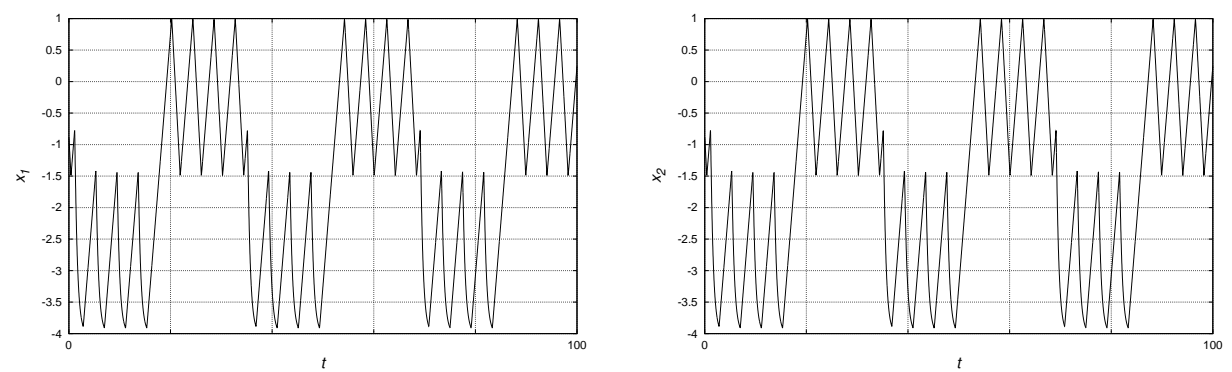

Рис. 13. Однородный режим с суммарно восемью промежутками положительности (l)
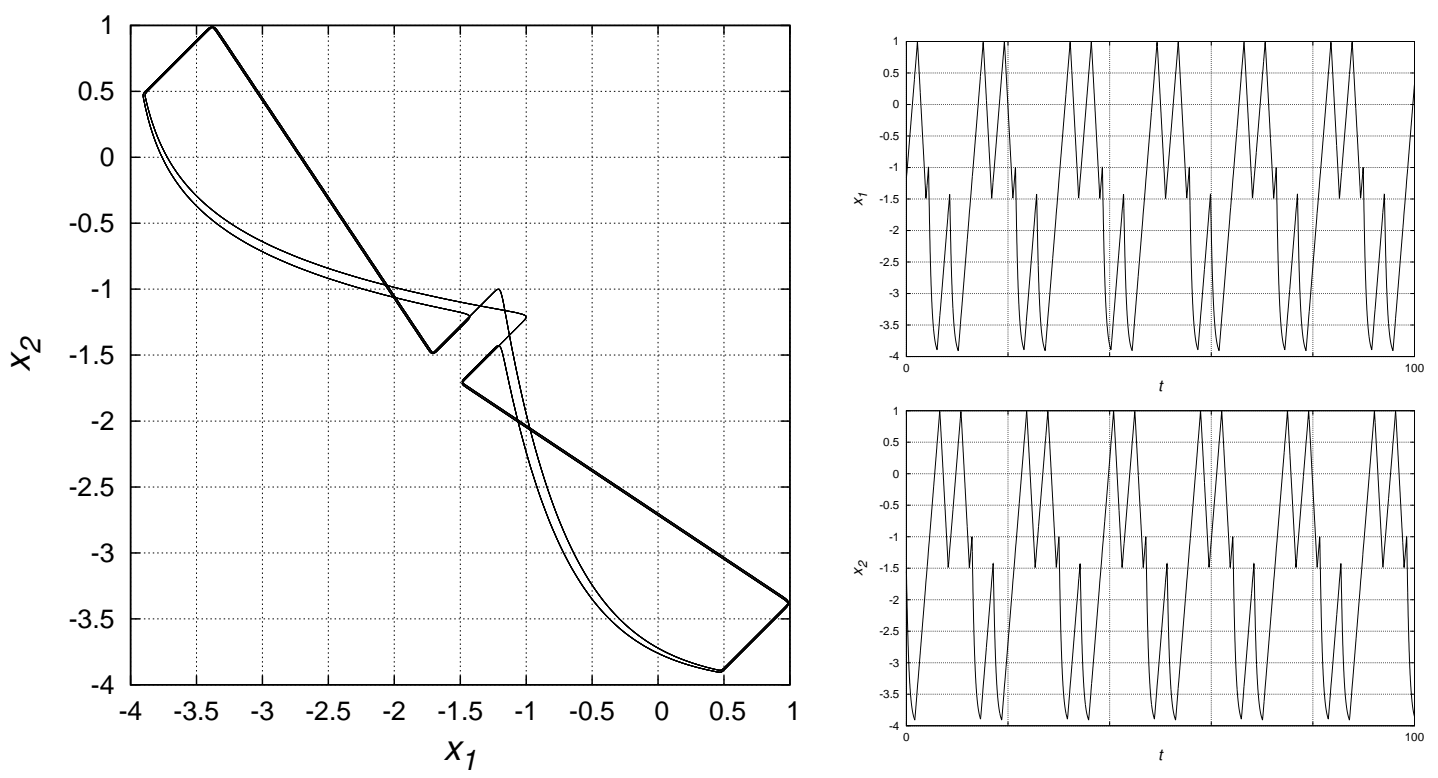

Рис. 14. Противофазный режим с суммарно четырьмя промежутками положительности (m)

близость к единичной окружности мультипликаторов $\mu_{2}$, описанных в лемме 3.2 , что указывает на наличие у этих режимов феномена квазиустойчивости. Режим (d) является импульсно-рефрактерным; отделенность от нуля второго показателя Ляпунова соответствует значениям мультипликаторов, описанным в лемме 2.1 .

Перейдем теперь к другому комплекту сосуществующих режимов, которые реализуются при $a=1,5, b=2, c=-15,13, h=15,92$ (см. рис. 6-9, таблицу 2). Все представленные режимы обладают суммарно восемью промежутками положительности, при этом режим (h) является однородным. Численные результаты подтверждают наличие феномена квазиустойчивости у всех режимов (e)-(h).

Все указанные сосуществующие режимы при $a=1,5, b=2, c=-4,5, h=15,9167$ (см. рис. 1014 , таблицу 3 ) обладают четным количеством суммарных промежутков положительности на периоде, причем у режимов (i)-(l) наблюдается по восемь суммарных промежутков положительности, а у противофазного режима $(\mathrm{m})$ - четыре суммарных промежутка положительности. Близость к нулю второго и третьего показателей Ляпунова у противофазного режима соответствует значениям мультипликаторов, описанным в лемме 2.3. Таким образом, у всех режимов (i)-(m) численно подтверждается феномен квазиустойчивости.

Рассмотрим значения параметров $a=1,5, b=2, c=-4,5, h=7,58333$ и сосуществующие режимы, им соответствующие (см. рис. 15-17, таблицу 4). Удалось получить режимы (n) и (о), 

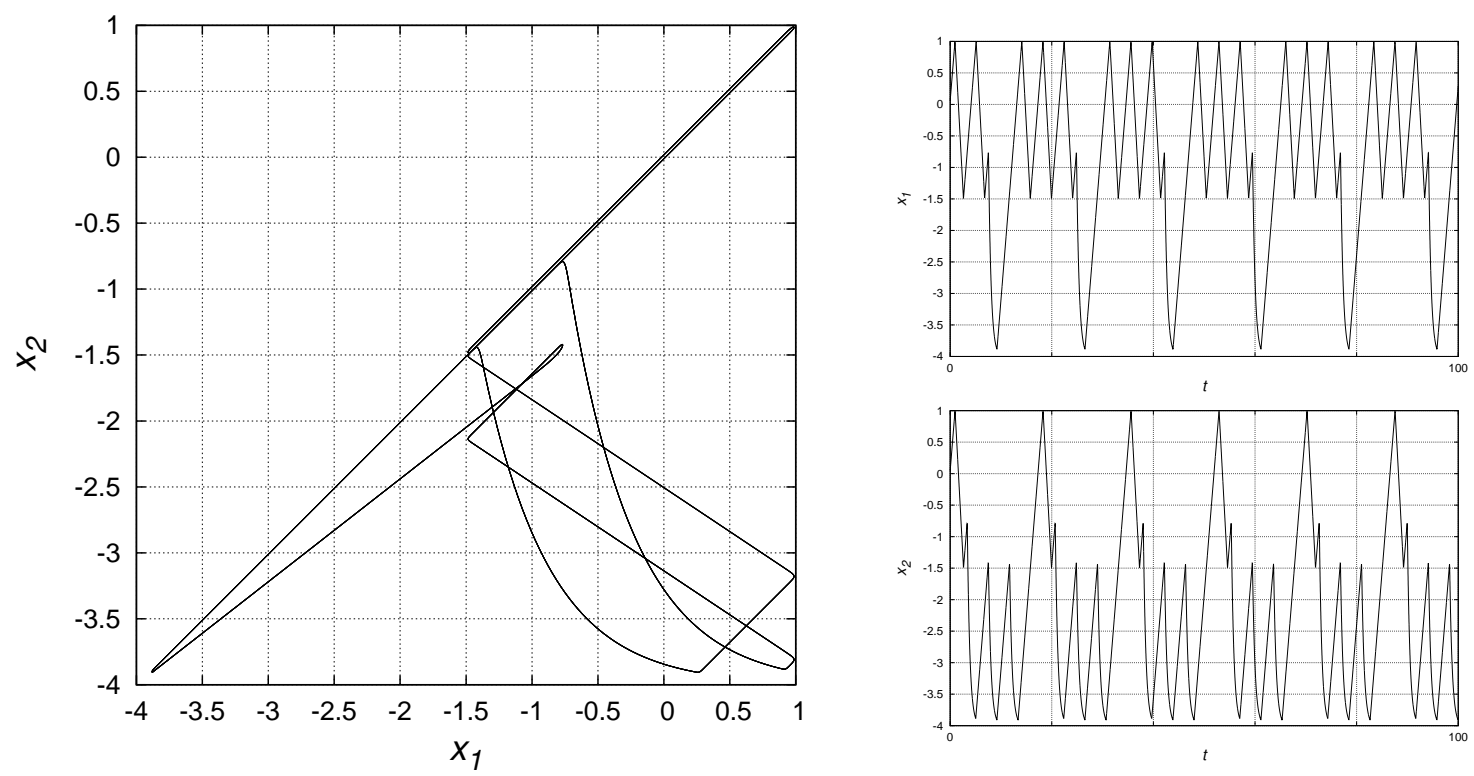

Рис. 15. Периодическое решение с тремя промежутками положительности первой компоненты и одним второй компоненты (n)

Таблица 2. Первые четыре показателя Ляпунова сосуществующих режимов (e)-(h) при $a=1,5, b=2, c=-15,13, h=15,92$

\begin{tabular}{|c|c|r|c|c|}
\hline & $\lambda_{1}$ & \multicolumn{1}{c|}{$\lambda_{2}$} & \multicolumn{1}{c|}{$\lambda_{3}$} & $\lambda_{4}$ \\
\hline (e) & 0,0000 & $-0,0007$ & $-0,8801$ & $-0,8842$ \\
(f) & 0,0000 & 0,0000 & $-0,8750$ & $-0,8842$ \\
(g) & 0,0000 & 0,0000 & $-0,8694$ & $-0,8807$ \\
(h) & 0,0000 & 0,0000 & $-0,8686$ & $-0,8820$ \\
\hline
\end{tabular}

Таблица 3. Первые четыре показателя Ляпунова сосуществующих режимов (i)-(m) при $a=1,5, b=2, c=-4,5, h=15,9167$

\begin{tabular}{|c|c|r|r|c|}
\hline & $\lambda_{1}$ & \multicolumn{1}{c|}{$\lambda_{2}$} & \multicolumn{1}{c|}{$\lambda_{3}$} & $\lambda_{4}$ \\
\hline$(\mathrm{i})$ & 0,0000 & $-0,0011$ & $-0,9167$ & $-0,9533$ \\
$(\mathrm{j})$ & 0,0000 & 0,0000 & $-0,9828$ & $-1,0417$ \\
$(\mathrm{k})$ & 0,0000 & 0,0000 & $-1,0422$ & $-1,0446$ \\
$(\mathrm{l})$ & 0,0000 & 0,0000 & $-1,0413$ & $-1,0443$ \\
$(\mathrm{~m})$ & 0,0000 & 0,0000 & 0,0000 & $-0,9570$ \\
\hline
\end{tabular}

причем режим (о) является однородным (суммарно у каждого из них по четыре суммарных промежутка положительности), а также противофазный режим (p), у которого лишь два промежутка положительности на периоде, при этом все представленные режимы обладают феноменом квазиустойчивости. 

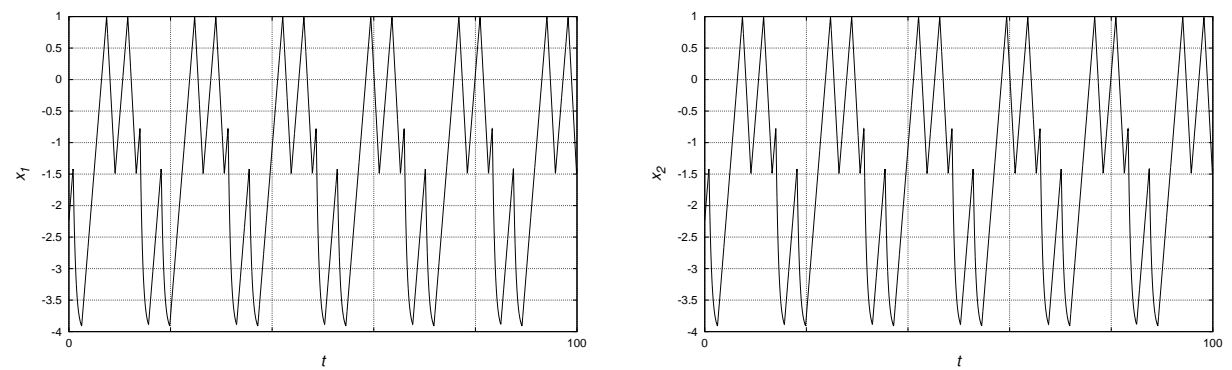

Рис. 16. Однородный режим с суммарно четырьмя промежутками положительности (о)
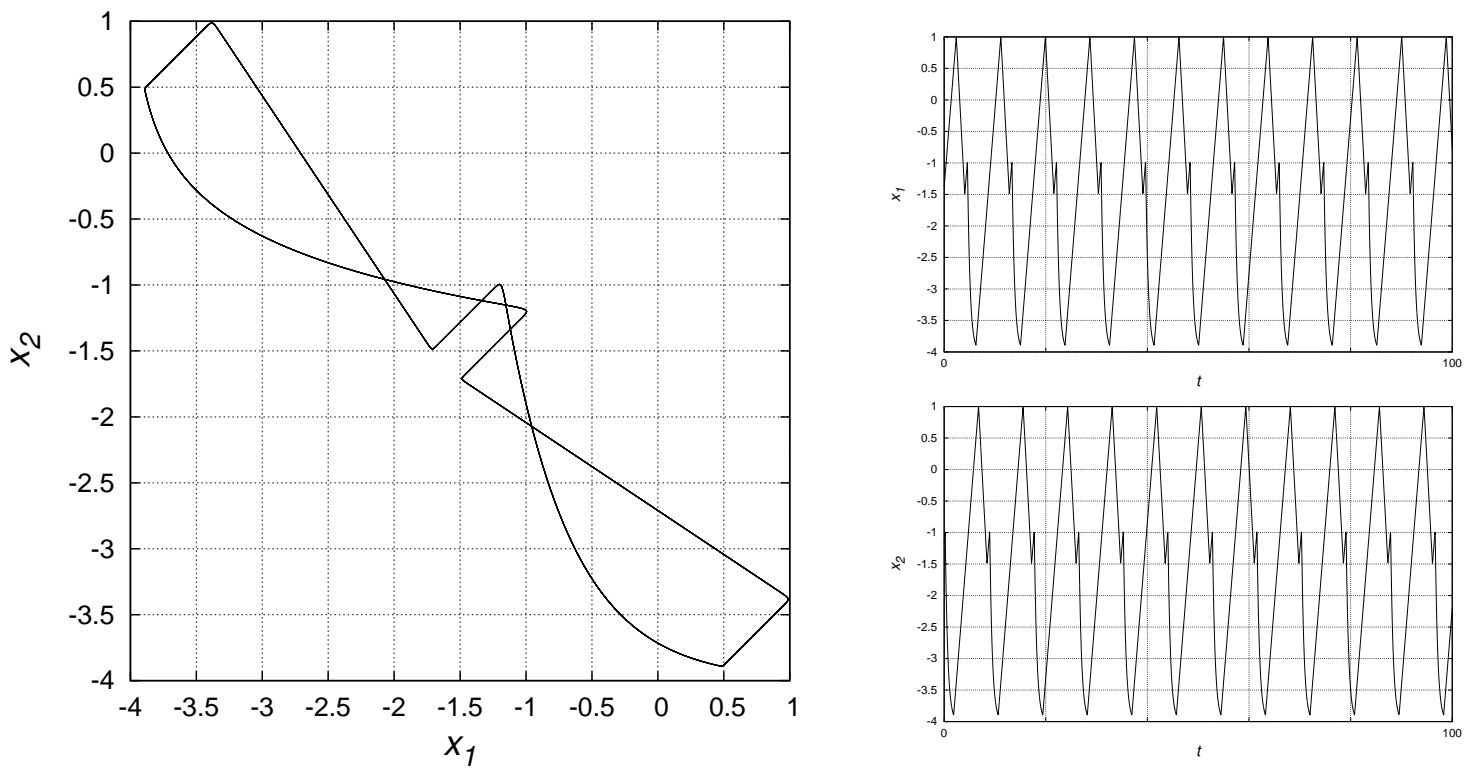

Рис. 17. Противофазный режим с суммарно двумя промежутками положительности (р)

Таблица 4. Первые четыре показателя Ляпунова сосуществующих режимов (n)-(p) при $a=1,5, b=2, c=-4,5, h=7,58333$

\begin{tabular}{|c|c|c|c|c|}
\hline & $\lambda_{1}$ & $\lambda_{2}$ & $\lambda_{3}$ & $\lambda_{4}$ \\
\hline$(\mathrm{n})$ & 0,0000 & $-0,0020$ & $-2,0710$ & $-2,0797$ \\
$(\mathrm{o})$ & 0,0000 & $-0,0001$ & $-2,0781$ & $-2,0857$ \\
(p) & 0,0000 & $-0,0041$ & $-0,0041$ & $-2,1927$ \\
\hline
\end{tabular}

Все рассмотренные выше режимы, за исключением импульсно-рефрактерного, обладают четным суммарным количеством промежутков положительности на периоде, что обусловлено специальным выбором параметров для соответствия представленным в этой работе аналитическим результатам. Но численно удается показать наличие феномена квазиустойчивости и у режимов схожей структуры с нечетным суммарным количеством промежутков положительности, например, при значениях параметров $a=1,5, b=2, c=-4,5, h=13,7$ (см. рис. $18-20$, таблицу 5). 

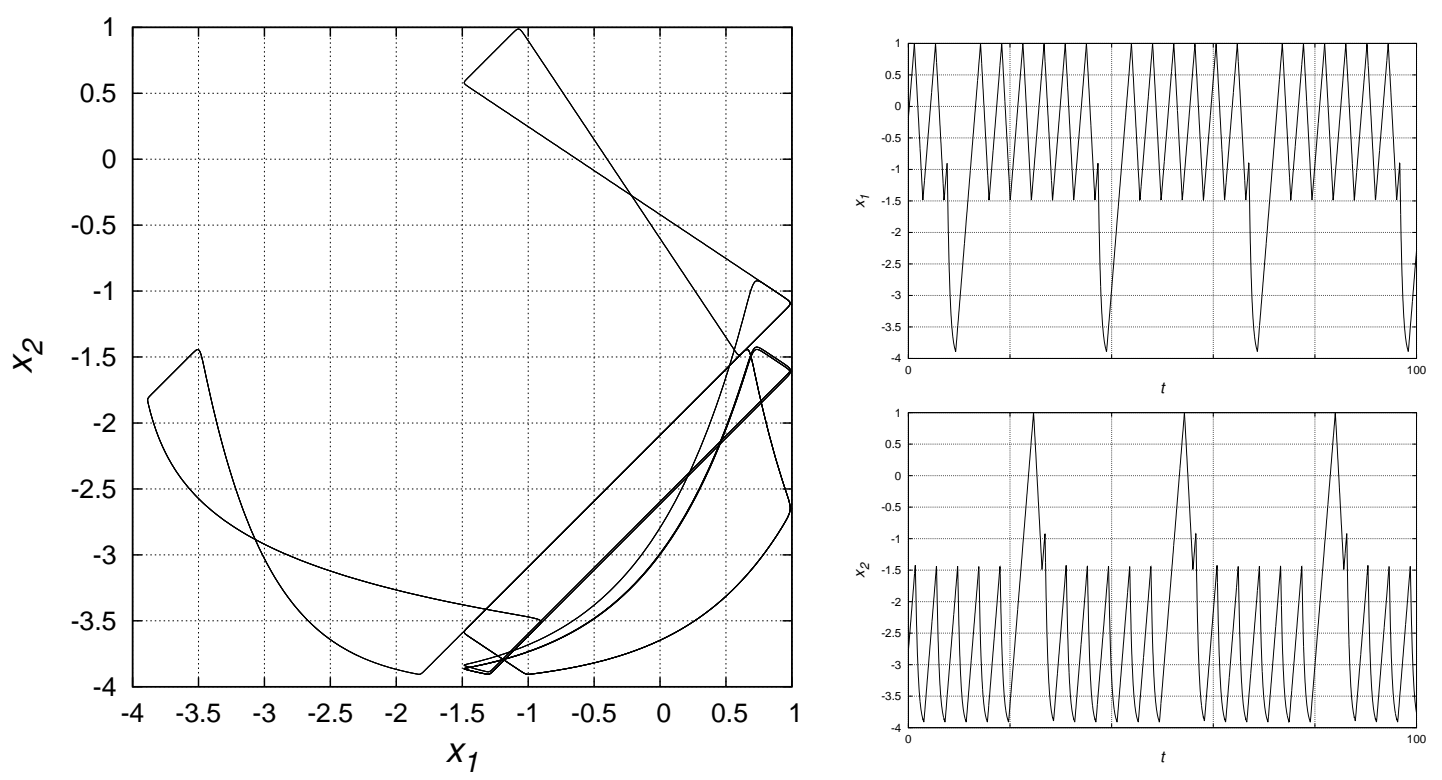

Рис. 18. Периодическое решение с шестью промежутками положительности первой компоненты и одним второй компоненты (q)

Таблица 5. Первые четыре показателя Ляпунова сосуществующих режимов (q)-(s) при $a=1,5, b=2, c=-4,5, h=13,7$

\begin{tabular}{|c|c|r|c|c|}
\hline & $\lambda_{1}$ & \multicolumn{1}{c|}{$\lambda_{2}$} & \multicolumn{1}{c|}{$\lambda_{3}$} & $\lambda_{4}$ \\
\hline (q) & 0,0000 & $-0,0012$ & $-1,0672$ & $-1,1220$ \\
$(\mathrm{r})$ & 0,0000 & 0,0000 & $-1,1962$ & $-1,1982$ \\
(s) & 0,0000 & 0,0000 & $-1,1975$ & $-1,2015$ \\
\hline
\end{tabular}

5. Заключение. Асимптотический анализ устойчивых релаксационных периодических режимов сингулярно возмущенной системы (1.1) и сопутствующая ему численная оценка ляпуновских показателей этих режимов подтвердили наличие у них эффекта квазиустойчивости. При этом, в отличие от статьи [9], где один из мультипликаторов задачи асимптотически близок к единичному значению, в нашем случае мультипликаторы оказываются близкими к единице не по причине наличия большого параметра, а в соответствии с выбором параметров задачи, каждый из которых имеет порядок единицы. Таким образом, вполне общей является ситуация, когда в динамической системе, на первый взгляд, вовсе нет малого или большого параметра, а переходный процесс к периодическому решению оказывается весьма длительным. При численном анализе таких систем вполне вероятна следующая ситуация. После определенного промежутка вычислений может показаться, что решение вышло на стационарный периодический режим. Вместе с тем более длительные вычисления могут привести к переходу к другому режиму, в связи с чем требуется вычислительный инструмент, который позволил бы эффективно диагностировать такие ситуации. Предложенный нами метод оценки спектра ляпуновских показателей как раз и служит таким инструментом. Ситуация, при которой близкими к нулю являются не только старший, но и еще какое-то количество ляпуновских показателей, свидетельствует о том, что либо устойчивый режим динамической системы является многочастотным, либо, как в нашем случае, реализуется явление квазиустойчивости. Знание этой дополнительной информации позволяет более эффективно применять численные методы к динамическим системам с запаздыванием. 

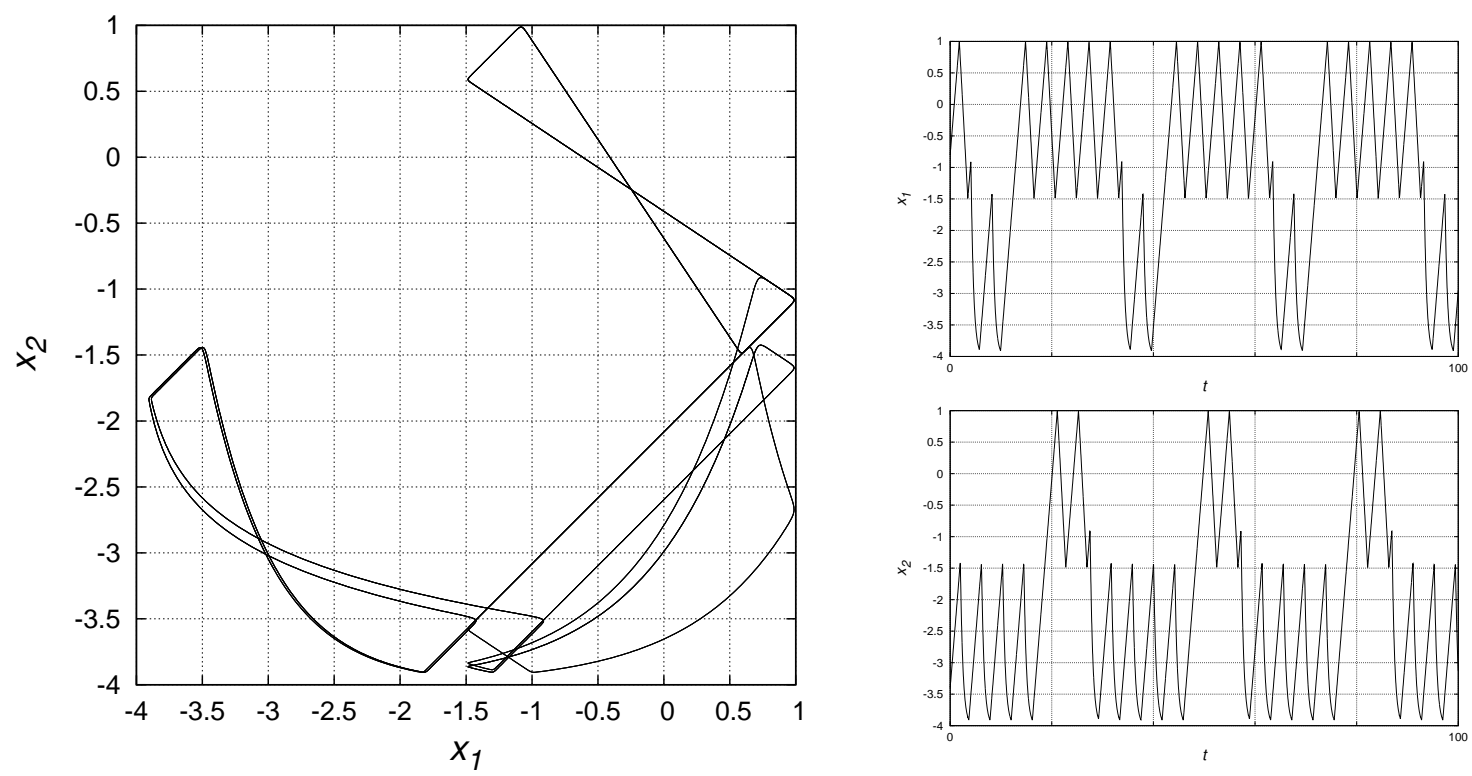

Рис. 19. Периодическое решение с пятью промежутками положительности первой компоненты и двумя второй компоненты (r)
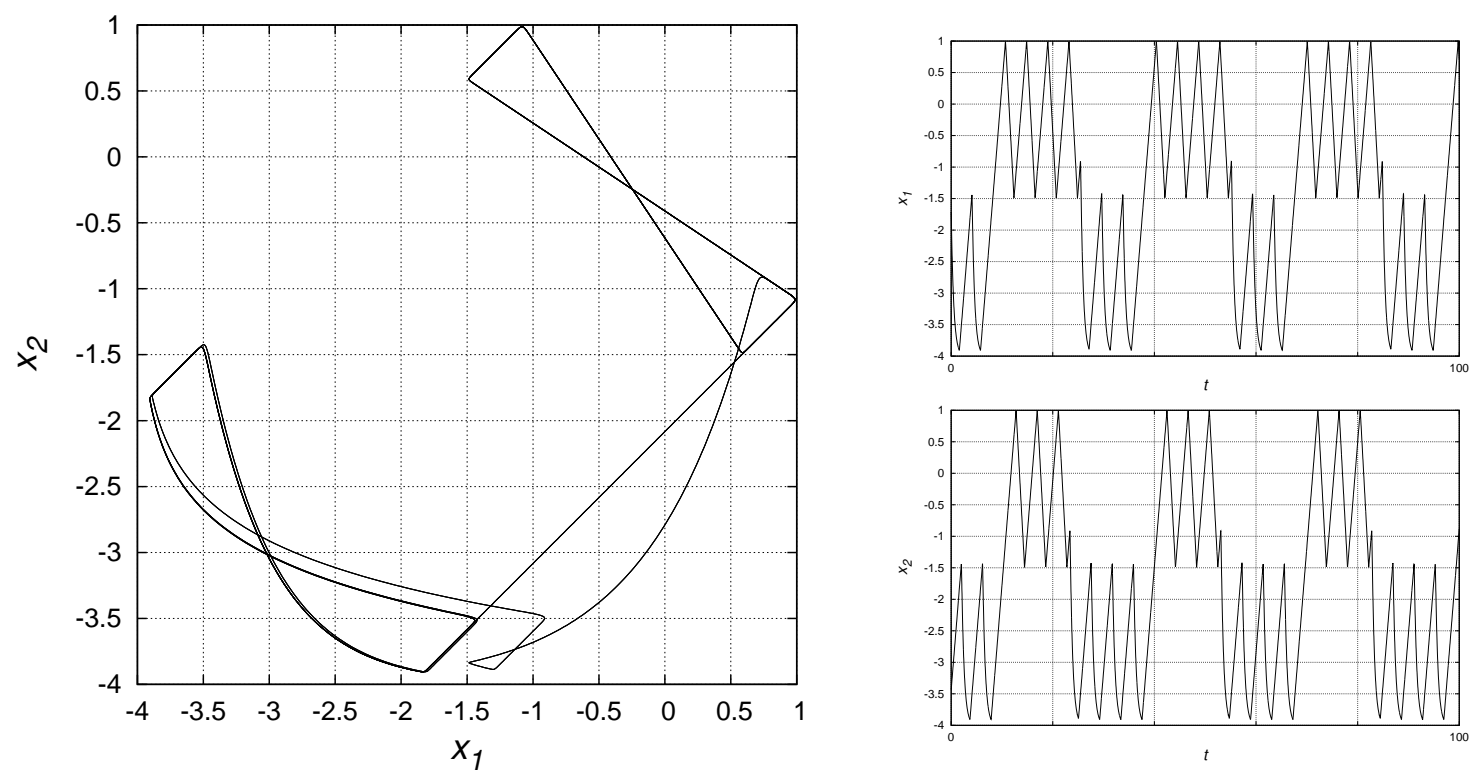

Рис. 20. Периодическое решение с четырьмя промежутками положительности первой компоненты и тремя второй компоненты (s)

Авторы выражают сердечную благодарность Сергею Дмитриевичу Глызину за постановку задачи и плодотворное обсуждение работы. 


\section{СПИСОК ЛИТЕРАТУРЫ}

1. Алешин C. В. Оценка инвариантных числовых показателей аттракторов систем дифференциальных уравнений с запаздыванием// в кн.: Вычислительные технологии в естественных науках (Назиров Р. Р., Щур Л. Н., ред.)/ Науч. конф. «Методы суперкомпьютерного моделирования» (Таруса, 1-3 октября 2014). - М.: Ин-т космич. исслед. РАН, 2014. - С. 10-17.

2. Былов Б. Ф., Виноград Р. Э., Гробман Д. М., Немъикий В. В. Теория показателей Ляпунова и ее приложения к вопросам устойчивости. - М.: Наука, 1966.

3. Глызин С. Д., Колесов А. Ю., Марушкина Е. А., Преображенскал М. М. Релаксационные автоколебания в системе из двух синаптически связанных импульсных нейронов// XIX Междунар. науч.-техн. конф. «Нейроинформатика-2017». Ч. 1 (Москва, 2-6 октября 2017). - М., 2017. - С. 29-39.

4. Глызин С. Д., Колесов А. Ю, Розов Н. Х. Дискретные автоволны в нейронных системах// Ж. вычисл. мат. мат. физ. - 2012. - 52, № 5. - С. 840-858.

5. Глызин С. Д., Колесов А. Ю., Розов Н. Х. Об одном способе математического моделирования химических синапсов // Диффер. уравн. - 2013. - 49, № 10. - С. 1227-1244.

6. Глызин С. Д., Колесов А. Ю, Розов Н. Х. Релаксационные автоколебания в сетях Хопфилда с запаздыванием// Изв. РАН. Сер. мат. - 2013. - 77, № 2. - С. 53-96.

7. Глызин С. Д., Колесов А. Ю., Розов Н. Х. Моделирование эффекта взрыва в нейронных системах// Мат. заметки. - 2013. - 93, № 5. - С. 684-701.

8. Глызин С. Д., Колесов А. Ю, Розов Н. Х. Релаксационные автоколебания в сетях импульсных нейронов// Усп. мат. наук. - 2015. - 70, № 3 (423). - С. 3-76.

9. Глызин С. Д., Колесов А. Ю., Розов Н. Х. Квазиустойчивые структуры в кольцевых генных сетях// Ж. вычисл. мат. мат. физ. - 2018. - 58, № 5. - С. $682-704$.

10. Кащенко С. А., Майоров В. В. Модели волновой памяти. - М.: Либроком, 2009.

11. Колесов А. Ю., Мищенко Е. Ф., Розов Н. Х. Реле с запаздыванием и его $C^{1}$-аппроксимация// Тр. Мат. ин-та им. В. А. Стеклова РАН. - 1997. - 216. - С. 126-153.

12. Колесов А. Ю., Мищенко Е. Ф., Розов Н. Х. Об одной модификации уравнения Хатчинсона// Ж. вычисл. мат. мат. физ. - 2010. - 50, № 12. - С. 2099-2112.

13. Оселедец В. И. Мультипликативная эргодическая теорема. Характеристические показатели Ляпунова динамических систем// Тр. Моск. мат. о-ва. - 1968. - 19. - С. 179-210.

14. Преображенская $M$. . Р. Релаксационные циклы в модели синаптически взаимодействующих осцилляторов// Модел. анал. информ. систем. - 2017. - 24, № 2. - С. 186-204.

15. Преображенскал $M$. М. Импульсно-рефрактерный режим в кольцевой цепи синаптически связанных осцилляторов нейронного типа// Модел. анал. информ. систем. - 2017. - 24, № 5. - С. 550-566.

16. Cheney W., Kincaid D. Linear Algebra: Theory and Applications. - Sudbury, Massachusetts: Jones and Bartlett, 2012.

17. Hairer E., Wanner G., Norsett S. P. Solving Ordinary Differential Equations. I. Nonstiff Problems. Berlin-Heidelberg: Springer-Verlag, 2008.

18. Preobrazhenskaya M. M. Multipliers of an antiphase solution in a system of two coupled nonlinear relaxation oscillators// J. Phys. Conf. Ser. — 2019. - 1163. — 012062.

19. Rabinovich M. I., Varona P., Selverston A. I., Abarbanel H. D. I. Dynamical principles in neuroscience// Rev. Mod. Phys. - 2006. - 78. - P. 1213-1265.

20. Somers D., Kopell N. Rapid synchronization through fast threshold modulation// Biol. Cybern. — 1993. - 68. - P. 393-407.

21. Somers D., Kopell N. Anti-phase solutions in relaxation oscillators coupled through excitatory interactions// J. Math. Biol. - 1995. - 33. - P. 261-280.

22. Wolf A., Swift J. B., Swinney H. L., Vastano J. A. Determining Lyapunov exponents from a time series// Phys. D. - 1985. - 16. - P. 285-317.

Горюнов Владимир Евгеньевич

Ярославский государственный университет им. П. Г. Демидова

E-mail: salkar@ya.ru

Преображенская Маргарита Михайловна

Ярославский государственный университет им. П. Г. Демидова

E-mail: rita.preo@gmail.com 